Pacific

Journal of

Mathematics

SPHERICAL NILPOTENT ORBITS IN POSITIVE CHARACTERISTIC

RUSSELL FOWLER AND GERHARD RÖHRLE

Volume $237 \quad$ No. 2

October 2008 


\title{
SPHERICAL NILPOTENT ORBITS IN POSITIVE CHARACTERISTIC
}

\author{
RUSSELl FOWLER AND GERHARD RÖHRLE
}

\begin{abstract}
Let $G$ be a connected reductive linear algebraic group defined over an algebraically closed field of characteristic $p$. Assume that $p$ is $\operatorname{good}$ for $G$. In this note we classify all the spherical nilpotent $G$-orbits in the Lie algebra of $G$. The classification is the same as in the characteristic zero case obtained by D. I. Panyushev [1994]: for $e$ a nilpotent element in the Lie algebra of $G$, the $G$-orbit $G \cdot e$ is spherical if and only if the height of $e$ is at most 3 .
\end{abstract}

\section{Introduction}

Let $G$ be a connected reductive linear algebraic group defined over an algebraically closed field $k$ of characteristic $p>0$. With the exception of Section 4.5, we assume throughout that $p$ is good for $G$ (see Section 2.1 for a definition).

A spherical $G$-variety $X$ is an (irreducible) algebraic $G$-variety on which a Borel subgroup $B$ of $G$ acts with a dense orbit. Homogeneous spherical $G$-varieties $G / H$, for $H$ a closed subgroup of $G$, are of particular interest. They include flag varieties (when $H$ is a parabolic subgroup of $G$ ) as well as symmetric spaces (when $H$ is the fixed point subgroup of an involutive automorphism of $G$ ). We refer the reader to [Brion 1987; 1995] for more information on spherical varieties and for their representation-theoretic significance. These varieties enjoy a remarkable property: a Borel subgroup of $G$ acts on a spherical $G$-variety only with a finite number of orbits. This fundamental result is due to M. Brion [1986] and É. B. Vinberg [1986] independently in characteristic 0, and to F. Knop [1995, 2.6] in arbitrary characteristic.

Let $\mathfrak{g}=\operatorname{Lie} G$ be the Lie algebra of $G$. The aim of this note is to classify the spherical nilpotent $G$-orbits in $\mathfrak{g}$. In case $k$ is of characteristic zero, this classification was obtained by D. I. Panyushev [1994]. The classification is the same in case the characteristic of $k$ is good for $G$ : for $e \in \mathfrak{g}$ nilpotent, $G \cdot e$ is spherical if and only if the height of $e$ is at most 3 (Theorem 3.38). The height of $e$ is the

MSC2000: primary 20G15, 14L30; secondary 17B50.

Keywords: spherical orbit, nilpotent orbit, associated cocharacter.

The first author acknowledges funding by the EPSRC. 
highest degree in the grading of $\mathfrak{g}$ afforded by a cocharacter of $G$ associated to $e$ (Definition 2.25).

The methods employed by Panyushev [1994] do not apply in positive characteristic, that is, parts of the argument are based on the concept of "stabilizers in general position"; it is unknown whether these exist generically in positive characteristic. Thus a different approach is needed to address the question in this case.

We briefly sketch the contents of the paper. In Section 2 we collect the preliminary results we require. In particular, we discuss the concepts of complexity and sphericity, and more specifically the question of complexity of homogeneous spaces. In Section 2.5 we recall the basic results of Kempf-Rousseau Theory and in Section 2.6 we recall the fundamental concepts of associated cocharacters for nilpotent elements from [Jantzen 2004, § 5] and [Premet 2003]. There we also recall the grading of $\mathfrak{g}$ afforded by a cocharacter associated to a given nilpotent element and define the notion of the height of a nilpotent element as the highest occurring degree of such a grading (see Definition 2.25). The complexity of fibre bundles is discussed in Section 2.7, which is crucial for the sequel. In particular, in Theorem 2.32 we show that the complexity of a fixed nilpotent orbit $G \cdot e$ is given by the complexity of a smaller reductive group acting on a linear space. Precisely, let $\lambda$ be a cocharacter of $G$, that is associated to $e$. Then $P_{\lambda}$ is the destabilizing parabolic subgroup $P(e)$ defined by $e$, in the sense of Geometric Invariant Theory. Moreover,

$$
L=C_{G}\left(\lambda\left(k^{*}\right)\right)
$$

is a Levi subgroup of $P(e)$. We show in Theorem 2.32 that the complexity of $G \cdot e$ equals the complexity of the action of $L$ on the subalgebra

$$
\bigoplus_{i \geqslant 2} \mathfrak{g}(i, \lambda)
$$

of $\mathfrak{g}$ where the grading

$$
\mathfrak{g}=\bigoplus_{i \in \mathbb{Z}} \mathfrak{g}(i, \lambda)
$$

is afforded by $\lambda$. In Section 2.8 we recall the concept of a weighted Dynkin diagram associated to a nilpotent orbit from [Carter 1985, § 5]. There we also present the classification of the parabolic subgroups $P$ of a simple algebraic group $G$ admitting a dense action of a Borel subgroup of a Levi subgroup of $P$ on the unipotent radical of $P$ according to [Brundan 1998, Theorem 4.1]. There we also remind the reader of the classification of the parabolic subgroups of $G$ with an abelian unipotent radical.

In Section 3 we give the classification of the spherical nilpotent orbits in good characteristic: a nilpotent element $e$ in $\mathfrak{g}$ is spherical if and only if the height of $e$ is at most 3 (Theorem 3.38). In Sections 3.1 and 3.3 we show that orbits of 
height 2 are spherical and orbits of height at least 4 are not, respectively. The remainder of Section 3 deals with the cases of height 3 nilpotent classes. For classical groups these only occur for the orthogonal groups. For the exceptional groups the height 3 cases are handled in Section 3.7 with the aid of a computer programme of S. M. Goodwin.

In Section 4 we discuss some further results and some applications of the classification. In Section 4.1 we discuss the spherical nilpotent orbits that are distinguished and in Section 4.2 we extend a result of Panyushev in characteristic zero to good positive characteristic: a characterization of the spherical nilpotent orbits in terms of pairwise orthogonal simple roots, see Theorem 4.14.

In Section 4.3 we discuss generalizations of results from [Panyushev and Röhrle $2001 ; 2005]$ to positive characteristic. In Theorem 4.18 we show that if $\mathfrak{a}$ is an abelian ideal of a Borel subalgebra $\mathfrak{b}$ of $\mathfrak{g}$, then $G \cdot \mathfrak{a}$ is a spherical variety. In Section 4.4 we describe a geometric characterization of spherical orbits in simple algebraic groups from [Cantarini et al. 2005] and [Carnovale 2006]. Finally, in Section 4.5 we very briefly touch on the issue of spherical nilpotent orbits in bad characteristic.

Thanks to the fact that a Springer isomorphism between the unipotent variety of $G$ and the nilpotent variety of $\mathfrak{g}$ affords a bijection between the unipotent $G$ classes in $G$ and the nilpotent $G$-orbits in $\mathfrak{g}$ (see [Springer and Steinberg 1970, III, 3.12] and [Bardsley and Richardson 1985, Corollary 9.3.4]), there is an analogous classification of the spherical unipotent conjugacy classes in $G$.

For results on algebraic groups we refer the reader to Borel's book [1991] and for information on nilpotent classes we cite Jantzen's monograph [2004].

\section{Preliminaries}

2.1. Notation. Let $H$ be a linear algebraic group defined over an algebraically closed field $k$. We denote the Lie algebra of $H$ by Lie $H$ or by $\mathfrak{h}$. We write $H^{\circ}$ for the identity component of $H$ and $Z(H)$ for the centre of $H$. The derived subgroup of $H$ is denoted by $\mathscr{D} H$ and we write rank $H$ for the dimension of a maximal torus of $H$. The unipotent radical of $H$ is denoted by $R_{u}(H)$. We say that $H$ is reductive provided $H^{\circ}$ is reductive. Let $K$ be a subgroup of $H$. We write $C_{H}(K)=\left\{h \in H \mid h x h^{-1}=x\right.$ for all $\left.x \in K\right\}$ for the centralizer of $K$ in $H$.

Suppose $H$ acts morphically on an algebraic variety $X$. Then we say that $X$ is an $H$-variety. Let $x \in X$. Then $H \cdot x$ denotes the $H$-orbit of $x$ in $X$ and $C_{H}(x)=$ $\{h \in H \mid h \cdot x=x$ for all $h \in H\}$ is the stabilizer of $x$ in $H$.

For $e \in \mathfrak{h}$ we denote the centralizers of $e$ in $H$ and $\mathfrak{h}$ by

$$
\begin{aligned}
C_{H}(e) & =\{h \in H \mid \operatorname{Ad}(h) e=e\}, \\
\mathfrak{c}_{\mathfrak{h}}(e) & =\{x \in \mathfrak{h} \mid[x, e]=0\} .
\end{aligned}
$$


For $S$ a subset of $H$ we write

$$
\mathfrak{c}_{\mathfrak{h}}(S)=\{x \in \mathfrak{h} \mid \operatorname{Ad}(s) x=x \text { for all } s \in S\}
$$

for the centralizer of $S$ in $\mathfrak{h}$.

Suppose $G$ is a connected reductive algebraic group. By $\mathcal{N}$ we denote the nilpotent cone of $\mathfrak{g}$. Let $T$ be a maximal torus of $G$. Let $\Psi=\Psi(G, T)$ denote the set of roots of $G$ with respect to $T$. Fix a Borel subgroup $B$ of $G$ containing $T$ and let $\Pi=\Pi(G, T)$ be the set of simple roots of $\Psi$ defined by $B$. Then $\Psi^{+}=\Psi(B, T)$ is the set of positive roots of $G$ with respect to $B$. For $I \subset \Pi$, we denote by $P_{I}$ and $L_{I}$ the standard parabolic and standard Levi subgroups of $G$ defined by $I$, respectively; see [Carter 1985, §2].

For $\beta \in \Psi^{+}$write

$$
\beta=\sum_{\alpha \in \Pi} c_{\alpha \beta} \alpha
$$

with $c_{\alpha \beta} \in \mathbb{N}_{0}$. A prime $p$ is said to be $\operatorname{good}$ for $G$ if it does not divide $c_{\alpha \beta}$ for any $\alpha$ and $\beta$ [Springer and Steinberg 1970, Definition 4.1]. Let $U=R_{u}(B)$ and set $\mathfrak{u}=$ Lie $U$. For a $T$-stable Lie subalgebra $\mathfrak{m}$ of $\mathfrak{u}$ we write

$$
\Psi(\mathfrak{m})=\left\{\beta \in \Psi^{+} \mid \mathfrak{g}_{\beta} \subseteq \mathfrak{m}\right\}
$$

for the set of roots of $\mathfrak{m}$ (with respect to $T$ ).

For every root $\beta \in \Psi$ we choose a generator $e_{\beta}$ for the corresponding root space $\mathfrak{g}_{\beta}$ of $\mathfrak{g}$. Any element $e \in \mathfrak{u}$ can be uniquely written as

$$
e=\sum_{\beta \in \Psi^{+}} c_{\beta} e_{\beta},
$$

where $c_{\beta} \in k$. The support of $e$ is defined as

$$
\operatorname{supp}(e)=\left\{\beta \in \Psi^{+} \mid c_{\beta} \neq 0\right\} .
$$

The variety of all Borel subgroups of $G$ is denoted by $\mathscr{B}$. Note that $\mathscr{B}$ is a single conjugacy class $\mathscr{B}=\left\{B^{g} \mid g \in G\right\}$. Also note the isomorphism $\mathscr{B} \cong G / B$.

Let $Y(G)=\operatorname{Hom}\left(k^{*}, G\right)$ be the set of cocharacters (one-parameter subgroups) of $G$. Likewise, for a closed subgroup $H$ of $G$, we set $Y(H)=\operatorname{Hom}\left(k^{*}, H\right)$ for the set of cocharacters of $H$. For $\lambda \in Y(G)$ and $g \in G$ we define $g \cdot \lambda \in Y(G)$ by

$$
(g \cdot \lambda)(t)=g \lambda(t) g^{-1}
$$

for $t \in k^{*}$; this gives a left action of $G$ on $Y(G)$. For $\mu \in Y(G)$ we write $C_{G}(\mu)$ for the centralizer of $\mu$ under this action of $G$ which coincides with $C_{G}\left(\mu\left(k^{*}\right)\right)$.

By a Levi subgroup of $G$ we mean a Levi subgroup of a parabolic subgroup of $G$. The Levi subgroups of $G$ are precisely the subgroups of $G$ which are of the form $C_{G}(S)$ where $S$ is a torus of $G$ [Borel 1991, Theorem 20.4]. Note that for $S$ a torus of $G$ the group $C_{G}(S)$ is connected [Borel 1991, Corollary 11.12]. 
2.2. Complexity. Suppose the linear algebraic group $H$ acts morphically on the (irreducible) algebraic variety $X$. Let $B$ be a Borel subgroup of $H$. Recall that the complexity of $X$ (with respect to the $H$-action on $X$ ) is defined as

$$
\kappa_{H}(X):=\min _{x \in X} \operatorname{codim}_{X} B \cdot x
$$

(see also [Brion 1995; Knop 1995; Luna and Vust 1983; Panyushev 1994; Vinberg 1986]).

Since the Borel subgroups of $H$ are conjugate in $H$ [Humphreys 1975, Theorem 21.3], the complexity of the variety $X$ is well defined.

Since a Borel subgroup of $H$ is connected, we have $\kappa_{H}(X)=\kappa_{H^{\circ}}(X)$. Thus for considering the complexity of an $H$-action, we may assume that $H$ is connected.

Concerning basic properties of complexity, we refer the reader to [Vinberg 1986, $\S 9]$.

We return to the general situation of a linear algebraic group $H$ acting on an algebraic variety $X$. For a Borel subgroup $B$ of $H$, we define

$$
\Gamma_{X}(B):=\left\{x \in X \mid \operatorname{codim}_{X} B \cdot x=\kappa_{H}(X)\right\} \subseteq X .
$$

Then we set

$$
\Gamma_{X}:=\bigcup_{B \in \mathscr{B}} \Gamma_{X}(B) \subseteq X
$$

For $x \in X$, we define

$$
\Lambda_{H}(x):=\left\{B \in \mathscr{B} \mid \operatorname{codim}_{X} B \cdot x=\kappa_{H}(X)\right\} \subseteq \mathscr{B} .
$$

Remark 2.1. The following statements are immediate from the definitions.

(i) If $H$ acts transitively on $X$, then $\Gamma_{X}=X$.

(ii) $B \in \Lambda_{H}(x)$ if and only if $x \in \Gamma_{X}(B)$.

(iii) $\Lambda_{H}(x)=\varnothing$ if and only if $x \notin \Gamma_{X}$.

The complexity of a reducible variety can easily be determined from the complexities of its irreducible components: Since a Borel subgroup $B$ of $G$ is connected, it stabilizes each irreducible component of $X$ [Humphreys 1975, Proposition 8.2(d)]. Let $x \in \Gamma_{X}(B)$ and choose an irreducible component $X^{\prime}$ of $X$ such that $x \in X^{\prime}$. Then $\kappa_{G}(X)=\kappa_{G}\left(X^{\prime}\right)+\operatorname{codim}_{X} X^{\prime}$. Therefore, from now on we may assume that $X$ is irreducible.

Next we recall from [Humphreys 1975, Proposition 4.4] that the dimension is upper semicontinuous.

Proposition 2.2. Let $\varphi: X \rightarrow Y$ be a dominant morphism of irreducible varieties. For $x \in X$, let $\varepsilon_{\varphi}(x)$ be the maximal dimension of any component of $\varphi^{-1}(\varphi(x))$ passing through $x$. Then $\left\{x \in X \mid \varepsilon_{\varphi}(x) \geqslant n\right\}$ is closed in $X$, for all $n \in \mathbb{Z}$. 
Corollary 2.3. Let $X$ be an $H$-variety. The set $\{x \in X \mid \operatorname{dim} H \cdot x \leqslant n\}$ is closed in $X$ for all $n \in \mathbb{Z}$. In particular, the union of all $H$-orbits of maximal dimension in $X$ is an open subset of $X$.

Lemma 2.4. For every $B \in \mathscr{B}$, we have $\Gamma_{X}(B)$ is a nonempty open subset of $X$.

Proof. Note that $\Gamma_{X}(B)$ is the union of $B$-orbits of maximal dimension. Thus, by Corollary 2.3, $\Gamma_{X}(B)$ is open in $X$.

Corollary 2.5. $\Gamma_{X}$ is open in $X$.

Next we need an easy but useful lemma; the proof is elementary.

Lemma 2.6. Let $\varphi: X \rightarrow Y$ be an $H$-equivariant dominant morphism of irreducible $H$-varieties. For $x \in X$, set $F_{\varphi(x)}=\varphi^{-1}(\varphi(x))$. Then $F_{\varphi(x)}$ is $C_{H}(\varphi(x))$ stable.

Before we can prove the next major result we need another preliminary:

Theorem 2.7 [Humphreys 1975, Theorem 4.3]. Let $\varphi: X \rightarrow Y$ be a dominant morphism of irreducible varieties. Set $r=\operatorname{dim} X-\operatorname{dim} Y$. Then there is a nonempty open subset $V$ of $Y$ such that $V \subseteq \varphi(X)$ and if $Y^{\prime} \subseteq Y$ is closed, irreducible and meets $V$ and $Z$ is a component of $\varphi^{-1}\left(Y^{\prime}\right)$ which meets $\varphi^{-1}(V)$, then $\operatorname{dim} Z=$ $\operatorname{dim} Y^{\prime}+r$. In particular, if $v \in V$, then $\operatorname{dim} \varphi^{-1}(v)=r$.

For the remainder of this section let $G$ be connected reductive. Let $\varphi: X \rightarrow Y$ be a $G$-equivariant dominant morphism of irreducible $G$-varieties. Then

$$
\kappa_{G}(Y) \leqslant \kappa_{G}(X)
$$

(see [Vinberg 1986, §9]). In our next result we give an interpretation for the difference $\kappa_{G}(X)-\kappa_{G}(Y)$ in terms of the complexity of a smaller subgroup acting on a fibre of $\varphi$.

Theorem 2.8. Let $\varphi: X \rightarrow Y$ be a G-equivariant dominant morphism of irreducible $G$-varieties. For $x \in X$ set $F_{\varphi(x)}=\varphi^{-1}(\varphi(x))$. Then for every $B \in \mathscr{B}$ there exists $x \in \Gamma_{X}(B)$ such that for $H=C_{B}(\varphi(x))^{\circ}$ we have

$$
\kappa_{G}(X)=\kappa_{G}(Y)+\kappa_{H}(Z),
$$

where $Z$ is an irreducible component of $F_{\varphi(x)}$ passing through $x$.

Proof. Let $B \in \mathscr{B}$. Let $V$ be a nonempty open subset of $Y$ which satisfies the conditions in Theorem 2.7. Since $Y$ is irreducible, Lemma 2.4 implies that

$$
\Gamma_{Y}(B) \cap V \neq \varnothing .
$$


For $y \in \Gamma_{Y}(B) \cap V$, Theorem 2.7 implies that any component of $\varphi^{-1}(y)$ has dimension $r=\operatorname{dim} X-\operatorname{dim} Y$, in particular, $\operatorname{dim} \varphi^{-1}(y)=r$. Since $\varphi^{-1}\left(\Gamma_{Y}(B) \cap V\right)$ is open in $X$, we have

$$
\varphi^{-1}\left(\Gamma_{Y}(B) \cap V\right) \cap \Gamma_{X}(B) \neq \varnothing,
$$

by Lemma 2.4. Now choose

$$
x \in \varphi^{-1}\left(\Gamma_{Y}(B) \cap V\right) \cap \Gamma_{X}(B) .
$$

In particular, $\operatorname{dim} F_{\varphi(x)}=r$. Lemma 2.6 implies that $F_{\varphi(x)}$ is $C_{B}(\varphi(x))$-stable. Clearly, $C_{B}(x)$ is the stabilizer of $x$ in $C_{B}(\varphi(x))$. Thus we obtain

$$
\begin{aligned}
\operatorname{codim}_{F_{\varphi(x)}} C_{B} & (\varphi(x)) \cdot x \\
& =\operatorname{dim} F_{\varphi(x)}-\operatorname{dim} C_{B}(\varphi(x)) \cdot x \\
& =r-\operatorname{dim} C_{B}(\varphi(x))+\operatorname{dim} C_{B}(x) \\
& =\operatorname{dim} X-\operatorname{dim} Y-\operatorname{dim} C_{B}(\varphi(x))+\operatorname{dim} C_{B}(x)+\operatorname{dim} B-\operatorname{dim} B \\
& =\operatorname{dim} X-\operatorname{dim} B+\operatorname{dim} C_{B}(x)-\left(\operatorname{dim} Y-\operatorname{dim} B+\operatorname{dim} C_{B}(\varphi(x))\right) \\
& =\kappa_{G}(X)-\kappa_{G}(Y),
\end{aligned}
$$

where the last equality holds because $x \in \Gamma_{X}(B)$ and $\varphi(x) \in \Gamma_{Y}(B)$.

Let $Z$ be an irreducible component of $F_{\varphi(x)}$ which passes through $x$. Theorem 2.7 implies that $Z$ has the same dimension as $F_{\varphi(x)}$. The connected group $H=C_{B}(\varphi(x))^{\circ}$ stabilizes $Z$. Note that for each $z \in Z$ we have $\varphi(z)=\varphi(x)$ and $C_{B}(z)=C_{C_{B}(\varphi(x))}(z)$ (observed for $z=x$ above). Since $x \in \Gamma_{X}(B), \operatorname{dim} C_{B}(x)$ is minimal among groups of the form $C_{B}(z)$ for $z \in Z$. Therefore, because $C_{B}(z)=$ $C_{C_{B}(\varphi(x))}(z)$, we see that $\operatorname{dim} C_{C_{B}(\varphi(x))}(x)$ is minimal among groups of the form $C_{C_{B}(\varphi(z))}(z)$ for $z \in Z$. We deduce that $x \in \Gamma_{Z}(H)$. Consequently,

$$
\begin{aligned}
\kappa_{H}(Z) & =\operatorname{dim} Z-\operatorname{dim} C_{B}(\varphi(x))^{\circ}+\operatorname{dim} C_{C_{B}(\varphi(x))^{\circ}}(x) \\
& =\operatorname{codim}_{F_{\varphi(x)}} C_{B}(\varphi(x)) \cdot x .
\end{aligned}
$$

2.3. Spherical varieties. A $G$-variety $X$ is called spherical if a Borel subgroup of $G$ acts on $X$ with a dense orbit, that is, $\kappa_{G}(X)=0$. We recall some standard facts concerning spherical varieties [Brion 1995; Knop 1995; Panyushev 1994].

First we recall an important result due to É. B. Vinberg [1986] and M. Brion [1986] independently in characteristic zero and F. Knop [1995, Corollary 2.6] in arbitrary characteristic. Let $B$ be a Borel subgroup of $G$.

Theorem 2.9. A spherical $G$-variety consists only of a finite number of B-orbits.

We have an immediate corollary. 
Corollary 2.10. The following are equivalent.

(i) The G-variety $X$ is spherical.

(ii) There is an open $B$-orbit in $X$.

(iii) The number of $B$-orbits in $X$ is finite.

2.4. Homogeneous spaces. Let $H$ be a closed subgroup of $G$. Since $G / H$ is a $G$-variety, we may consider the complexity $\kappa_{G}(G / H)$. Let $B$ be a Borel subgroup of $G$. The orbits of $B$ on $G / H$ are in bijection with the $(B, H)$-double cosets of $G$. We have that

$$
\kappa_{G}(G / H)=\operatorname{codim}_{G / H} B g H / H
$$

for $g H \in \Gamma_{G / H}(B)$. Clearly, $G$ acts transitively on $G / H$, so Remark 2.1(i) implies that we can choose a Borel subgroup $B$ such that $B \in \Lambda_{G}(1 H)$. Thus, for this choice of $B$, we have

$$
\begin{aligned}
\kappa_{G}(G / H) & =\operatorname{codim}_{G / H} B H / H=\operatorname{dim} G / H-\operatorname{dim} B H / H \\
& =\operatorname{dim} G / H-\operatorname{dim} B / B \cap H \\
& =\operatorname{dim} G-\operatorname{dim} H-\operatorname{dim} B+\operatorname{dim} B \cap H .
\end{aligned}
$$

Following M. Krämer [1979], a subgroup $H$ of $G$ is called spherical if

$$
\kappa_{G}(G / H)=0 .
$$

Since $\kappa_{G}(G / H)=\kappa_{G}\left(G / H^{\circ}\right)$, by (2-1), in considering the complexity of homogeneous spaces $G / H$ we may assume that the subgroup $H$ is connected.

We have an easy lemma.

Lemma 2.11. Let $G$ be connected reductive and let $H$ be a subgroup of $G$ which contains the unipotent radical of a Borel subgroup of $G$. Then $H$ is spherical. In particular, a parabolic subgroup of $G$ is spherical.

Proof. Let $B$ be a Borel subgroup of $G$ such that $U=R_{u}(B) \leqslant H$. Denote by $B^{-}$ the opposite Borel subgroup to $B$, relative to some maximal torus of $B$ [Humphreys $1975, \S 26.2$, Corollary C]. The big cell $B^{-} U$ is an open subset of $G$ [Humphreys 1975, Proposition 28.5]. We have $B^{-} U \subseteq B^{-} H$, so $B^{-} H$ is a dense subset of $G$. Thus, $G / H$ is spherical.

Remark 2.12. If both $G$ and $H$ are reductive, then $G / H$ is an affine variety [Richardson 1977, Theorem A]. This case has been studied greatly. The classification of spherical reductive subgroups of the simple algebraic groups in characteristic zero was obtained by M. Krämer [1979] and was shown to be the same in positive characteristic by J. Brundan [1998]. M. Brion [1987] classifies all the spherical reductive subgroups of an arbitrary reductive group in characteristic zero. 
In positive characteristic no such classification is known. However, the classification of the reductive spherical subgroups in simple algebraic groups in positive characteristic follows from the work of T. A. Springer [1985] (see also [Seitz 1998; Brundan 1998; Lawther 1999]).

Important examples of reductive spherical subgroups are centralizers of involutive automorphisms of $G$ : Suppose that char $k \neq 2$ and let $\theta$ be an involutive automorphism of $G$. Then the fixed point subgroup $C_{G}(\theta)=\{g \in G \mid \theta(g)=g\}$ of $G$ is spherical [Springer 1985, Corollary 4.3.1].

For more on the complexity and sphericity of homogeneous spaces see [Brion 1986; Luna and Vust 1983; Panyushev 1990].

Remark 2.13. In order to compute the complexity of an orbit variety, it suffices to determine the complexity of a homogeneous space. Suppose that $G$ acts on an algebraic variety $X$. Let $x \in X$. Since $G$ is connected, the orbit $G \cdot x$ is irreducible. The map

$$
\pi_{x}: G / C_{G}(x) \rightarrow G \cdot x, \quad \pi_{x}\left(g C_{G}(x)\right)=g \cdot x
$$

is a bijective $G$-equivariant morphism [Jantzen 2004, §2.1]. Thus, by applying Theorem 2.8 to $\pi_{x}$, we have

$$
\kappa_{G}\left(G / C_{G}(x)\right)=\kappa_{G}(G \cdot x) .
$$

The relevance of (2-2) is that the left hand side is easier to compute, since calculating $\kappa_{G}\left(G / C_{G}(x)\right)$ only requires the study of groups of the form $C_{B}(x)$, where $B$ is a Borel subgroup of $G$ (compare (2-1)).

2.5. Kempf-Rousseau Theory. Next we require some standard facts from Geometric Invariant Theory [Kempf 1978] (also see [Premet 2003, §2] and [Richardson $1982, \S 7])$. Let $X$ be an affine variety and $\phi: k^{*} \rightarrow X$ be a morphism of algebraic varieties. We say that $\lim _{t \rightarrow 0} \phi(t)$ exists if there exists a morphism $\widehat{\phi}: k \rightarrow X$ such that $\left.\widehat{\phi}\right|_{k^{*}}=\phi$. If such a limit exists, we set

$$
\lim _{t \rightarrow 0} \phi(t)=\widehat{\phi}(0) \text {. }
$$

Note that if such a morphism $\widehat{\phi}$ exists, it is necessarily unique.

Let $\lambda$ be a cocharacter of $G$. Define

$$
P_{\lambda}=\left\{x \in G \mid \lim _{t \rightarrow 0} \lambda(t) x \lambda(t)^{-1} \text { exists }\right\} .
$$

Then $P_{\lambda}$ is a parabolic subgroup of $G$, the unipotent radical of $P_{\lambda}$ is given by

$$
R_{u}\left(P_{\lambda}\right)=\left\{x \in G \mid \lim _{t \rightarrow 0} \lambda(t) x \lambda(t)^{-1}=1\right\},
$$


and a Levi subgroup of $P_{\lambda}$ is the centralizer

$$
G_{G}(\lambda)=C_{G}\left(\lambda\left(k^{*}\right)\right)
$$

of the image of $\lambda$ in $G$ [Springer 1998, §8.4].

Let the connected reductive group $G$ act on the affine variety $X$ and suppose $x \in X$ is a point such that $G \cdot x$ is not closed in $X$. Let $C$ denote the unique closed $G$-orbit in the closure of $G \cdot x$ (see [Richardson 1977, Lemma 1.4]). Set

$$
\Lambda(x):=\left\{\lambda \in Y(G) \mid \lim _{t \rightarrow 0} \lambda(t) \cdot x \text { exists and lies in } C\right\} .
$$

Then there is a so-called optimal class $\Omega(x) \subseteq \Lambda(x)$ of cocharacters associated to $x$. The following theorem is due to G. R. Kempf [1978, Theorem 3.4] (see also [Rousseau 1978]).

Theorem 2.14. Assume as above. Then we have the following:

(i) $\Omega(x) \neq \varnothing$.

(ii) There exists a parabolic subgroup $P(x)$ of $G$ such that $P(x)=P_{\lambda}$ for every $\lambda \in \Omega(x)$.

(iii) $\Omega(x)$ is a single $P(x)$-orbit.

(iv) For $g \in G$, we have $\Omega(g \cdot x)=g \cdot \Omega(x)$ and $P(g \cdot x)=g P(x) g^{-1}$. In particular, $C_{G}(x) \leqslant N_{G}(P(x))=P(x)$.

Frequently, $P(x)$ in Theorem 2.14 is called the destabilizing parabolic subgroup of $G$ defined by $x \in X$.

2.6. Associated cocharacters. Here we closely follow [Premet 2003] (also see [Jantzen 2004, §5]). We recall that $p$ is a good prime for $G$ throughout this section.

Every cocharacter $\lambda \in Y(G)$ induces a grading of $\mathfrak{g}$ :

$$
\mathfrak{g}=\bigoplus_{i \in \mathbb{Z}} \mathfrak{g}(i, \lambda)
$$

where

$$
\mathfrak{g}(i, \lambda)=\left\{x \in \mathfrak{g} \mid \operatorname{Ad}(\lambda(t))(x)=t^{i} x \text { for all } t \in k^{*}\right\}
$$

(see [Jantzen 2004, §5.1]). For $P_{\lambda}$ as in Section 2.5, we have the following equalities:

Lie $P_{\lambda}=\bigoplus_{i \geqslant 0} \mathfrak{g}(i, \lambda)$, Lie $R_{u}\left(P_{\lambda}\right)=\bigoplus_{i>0} \mathfrak{g}(i, \lambda)$, and Lie $C_{G}(\lambda)=\mathfrak{g}(0, \lambda)$

Frequently, we write $\mathfrak{g}(i)$ for $\mathfrak{g}(i, \lambda)$ once we have fixed a cocharacter $\lambda \in Y(G)$.

Let $H$ be a connected reductive subgroup of $G$. A nilpotent element $e \in \mathfrak{h}$ is called distinguished in $\mathfrak{h}$ provided each torus in $C_{H}(e)$ is contained in the centre of $H$ [Jantzen 2004, §4.1]. 
The following characterization of distinguished nilpotent elements in the Lie algebra of a Levi subgroup of $G$ can be found in [Jantzen 2004, §4.6, §4.7].

Proposition 2.15. Let $e \in \mathfrak{g}$ be nilpotent and let $L$ be a Levi subgroup of $G$. Then $e$ is distinguished in Lie $L$ if and only if $L=C_{G}(S)$, where $S$ is a maximal torus of $C_{G}(e)$.

Next we recall the definition of an associated cocharacter [Jantzen 2004, §5.3].

Definition 2.16. A cocharacter $\lambda: k^{*} \rightarrow G$ is associated to $e \in \mathcal{N}$ if $e \in \mathfrak{g}(2, \lambda)$ and there exists a Levi subgroup $L$ of $G$ such that $e$ is distinguished in Lie $L$, and $\lambda\left(k^{*}\right) \leqslant \mathscr{D} L$.

Remark 2.17. In view of Proposition 2.15, the last two conditions in Definition 2.16 are equivalent to the existence of a maximal torus $S$ of $C_{G}(e)$ such that $\lambda\left(k^{*}\right) \leqslant$ $\mathscr{D C} C_{G}(S)$. We will use this fact frequently in the sequel.

Let $e \in \mathcal{N}$. A. Premet [2003, §2.4, Proposition 2.5] explicitly defines a cocharacter of $G$ which is associated to $e$. Moreover, Premet [2003, Theorem 2.3] shows that each of these associated cocharacters belongs to the optimal class $\Omega(e)$ determined by $e$. He shows this under the so-called standard hypotheses on $G$; see [Jantzen 2004, §2.9]. These restrictions were subsequently removed by G. McNinch [2004, Proposition 16] so that this fact holds for any connected reductive group $G$ in good characteristic. It thus follows from [McNinch 2004, Proposition 16], Theorem 2.14(iv), and the fact that any two associated cocharacters are conjugate under $C_{G}(e)$, [Jantzen 2004, Lem. 5.3], that all the cocharacters of $G$ associated to $e \in \mathcal{N}$ belong to the optimal class $\Omega(e)$ defined by $e$ (see also [McNinch 2004, Proposition 18, Theorem 21]). This motivates and justifies the following notation which we use in the sequel.

Definition 2.18. Let $e \in \mathfrak{g}$ be nilpotent. Then we denote the set of cocharacters of $G$ associated to $e$ by

$$
\Omega_{G}^{a}(e):=\{\lambda \in Y(G) \mid \lambda \text { is associated to } e\} \subseteq \Omega(e) .
$$

Further, if $H$ is a (connected) reductive subgroup of $G$ with $e \in \mathfrak{h}$ nilpotent we also write $\Omega_{H}^{a}(e)$ to denote the cocharacters of $H$ that are associated to $e$.

As indicated above, in good characteristic, associated cocharacters are known to exist for any nilpotent element $e \in \mathfrak{g}$; more precisely, we have the following (see [Jantzen 2004, §5.3]):

Proposition 2.19. Suppose that $p$ is good for $G$. Let $e \in \mathfrak{g}$ be nilpotent. Then $\Omega_{G}^{a}(e) \neq \varnothing$. Moreover, if $\lambda \in \Omega_{G}^{a}(e)$ and $\mu \in Y(G)$, then $\mu \in \Omega_{G}^{a}(e)$ if and only if $\mu$ and $\lambda$ are conjugate by an element of $C_{G}(e)$. 
Fix a nilpotent element $e \in \mathfrak{g}$ and an associated cocharacter $\lambda \in \Omega_{G}^{a}(e)$ of $G$. Set $P=P_{\lambda}$. By Theorem 2.14(ii), $P$ only depends on $e$ and not on the choice of the associated cocharacter $\lambda$. Note that $C_{G}(\lambda)$ stabilizes $\mathfrak{g}(i)$ for every $i \in \mathbb{Z}$. For $n \in \mathbb{Z}_{\geqslant 0}$ we set

$$
\mathfrak{g}_{\geqslant n}=\bigoplus_{i \geqslant n} \mathfrak{g}(i) \quad \text { and } \quad \mathfrak{g}_{>n}=\bigoplus_{i>n} \mathfrak{g}(i)
$$

Then we have

$$
\mathfrak{g}_{\geqslant 0}=\text { Lie } P \quad \text { and } \quad \mathfrak{g}_{>0}=\operatorname{Lie} R_{u}(P) .
$$

Also, $C_{G}(e)=C_{P}(e)$, by Theorem 2.14(iv).

The next result is [Jantzen 2004, Proposition 5.9(c)].

Proposition 2.20. The P-orbit of $e$ in $\mathfrak{g}_{\geqslant 2}$ is dense in $\mathfrak{g} \geqslant 2$.

Corollary 2.21. The $C_{G}(\lambda)$-orbit of $e$ in $\mathfrak{g}(2)$ is dense in $\mathfrak{g}(2)$.

Definition 2.22. Let $e \in \mathcal{N}$ and let $\lambda \in \Omega_{G}^{a}(e)$. Then set

$$
C_{G}(e, \lambda):=C_{G}(e) \cap C_{G}(\lambda) .
$$

Corollary 2.23. Let $e \in \mathcal{N}$. Then

(i) $\operatorname{dim} C_{G}(e)=\operatorname{dim} \mathfrak{g}(0)+\operatorname{dim} \mathfrak{g}(1)$,

(ii) $\operatorname{dim} R_{u}\left(C_{G}(e)\right)=\operatorname{dim} \mathfrak{g}(1)+\operatorname{dim} \mathfrak{g}(2)$, and

(iii) $\operatorname{dim} C_{G}(e, \lambda)=\operatorname{dim} \mathfrak{g}(0)-\operatorname{dim} \mathfrak{g}(2)$.

Proof. As $C_{G}(e)=C_{P}(e)$, part (i) is immediate from Proposition 2.20. Using the fact that

$$
\operatorname{Ad}\left(R_{u}(P)-1\right)(e) \subseteq \mathfrak{g}_{\geqslant 3}
$$

(see [Jantzen 2004, §5.10]) and Proposition 2.20, we see that

$$
\operatorname{dim} \operatorname{Ad}\left(R_{u}(P)\right)(e)=\operatorname{dim} \mathfrak{g}_{\geqslant 3}
$$

and so

$$
\operatorname{dim} C_{R_{u}(P)}(e)=\operatorname{dim} \mathfrak{g}(1)+\operatorname{dim} \mathfrak{g}(2) .
$$

Finally, part (iii) follows from the first two.

The following basic result regarding the structure of $C_{G}(e)$ can be found in [Premet 2003, Theorem A].

Proposition 2.24. If char $k$ is good for $G$, then $C_{G}(e)$ is the semidirect product of $C_{G}(e, \lambda)$ and $C_{G}(e) \cap R_{u}(P)$. Moreover, $C_{G}(e, \lambda)^{\circ}$ is reductive and $C_{G}(e) \cap R_{u}(P)$ is the unipotent radical of $C_{G}(e)$. 
Definition 2.25. Let $e \in \mathfrak{g}$ be nilpotent. The height of $e$ with respect to an associated cocharacter $\lambda \in \Omega_{G}^{a}(e)$ is defined to be

$$
\operatorname{ht}(e):=\max _{i \in \mathbb{N}}\{i \mid \mathfrak{g}(i, \lambda) \neq 0\} .
$$

Thanks to Proposition 2.19, the height of $e$ does not depend on the choice of $\lambda \in$ $\Omega_{G}^{a}(e)$. Since conjugate nilpotent elements have the same height, we may speak of the height of a given nilpotent orbit. Since $\lambda \in \Omega_{G}^{a}(e)$, we have ht $(e) \geqslant 2$ for any nilpotent element $e \in \mathfrak{g}$; see Definition 2.16.

Let $\mathfrak{g}$ be classical with natural module $V$. Set $n=\operatorname{dim} V$. We write a partition $\pi$ of $n$ in one of the following two ways:

(i) $\pi=\left(d_{1}, d_{2}, \ldots, d_{r}\right)$ with $d_{1} \geqslant d_{2} \geqslant \cdots \geqslant d_{r} \geqslant 0$ and $\sum_{i=1}^{r} d_{i}=n$, or

(ii) $\pi=\left[1^{r_{1}}, 2^{r_{2}}, \ldots\right]$ with $\sum_{i} i r_{i}=n$.

These two notations are related by $r_{i}=\left|\left\{j \mid d_{j}=i\right\}\right|$ for $i \geqslant 1$.

For $\mathfrak{g}$ classical with natural module $V$, it is straightforward to determine the height of a nilpotent orbit from the corresponding partition of $\operatorname{dim} V$. We leave the proof of the next proposition to the reader.

Proposition 2.26. Let $e \in \mathfrak{g}$ be nilpotent with partition $\pi_{e}=\left(d_{1}, d_{2}, \ldots, d_{r}\right)$.

(i) If $\mathfrak{g}=\mathfrak{g l}(V), \mathfrak{s l}(V)$ or $\mathfrak{s p}(V)$, then $\mathrm{ht}(e)=2\left(d_{1}-1\right)$.

(ii) If $\mathfrak{g}=\mathfrak{s o}(V)$, then $\mathrm{ht}(e)= \begin{cases}2\left(d_{1}-1\right) & \text { if } d_{1}=d_{2}, \\ 2 d_{1}-3 & \text { if } d_{1}=d_{2}+1, \\ 2\left(d_{1}-2\right) & \text { if } d_{1}>d_{2}+1\end{cases}$

Remarks 2.27. (i) For char $k=0$, Proposition 2.26 was proved in [Panyushev 1999, Theorem 2.3].

(ii) If $e$ is a nilpotent element in $\mathfrak{g l}(V), \mathfrak{s l}(V)$ or $\mathfrak{s p}(V)$, then ht( $(e)$ is even. If $e$ is a nilpotent element in $\mathfrak{s o}(V)$, then ht $(e)$ is odd if and only if $d_{2}=d_{1}-1$.

2.7. Fibre bundles. Let $H$ be a closed subgroup of $G$. Suppose that $H$ acts on an affine variety $Y$. Define a morphic action of $H$ on the affine variety $G \times Y$ by

$$
h \cdot(g, y)=\left(g h, h^{-1} \cdot y\right)
$$

for $h \in H, g \in G$ and $y \in Y$. Since $H$ acts fixed point freely on $G \times Y$, every $H$ orbit in $G \times Y$ has dimension $\operatorname{dim} H$. There exists a surjective quotient morphism $\rho: G \times Y \rightarrow(G \times Y) / H$ (see [Mumford and Fogarty 1982, §1.2] and [Parshin and Shafarevich 1994, §4.8]). We denote the quotient $(G \times Y) / H$ by $G *_{H} Y$, the fibre bundle associated to the principal bundle $\pi: G \rightarrow G / H$ defined by $\pi(g)=g H$ and fibre $Y$. We denote the element $(g, y) H$ of $G *_{H} Y$ simply by $g * y$ [Richardson $1967, \S 2]$. Let $X$ be a $G$-variety and $Y \subseteq X$ be an $H$-subvariety. The collapsing of the fibre bundle $G *_{H} Y$ is the morphism $G *_{H} Y \rightarrow G \cdot Y \subseteq X$ defined by $g * y \rightarrow g \cdot y$. 
Define an action of $G$ on $G *_{H} Y$ by $g \cdot\left(g^{\prime} * y\right)=\left(g g^{\prime}\right) * y$ for $g, g^{\prime} \in G$ and $y \in Y$. We then have a $G$-equivariant surjective morphism $\varphi: G *_{H} Y \rightarrow G / H$ by $\varphi(g * y)=g H$. Note that $\varphi^{-1}(g H) \cong Y$ for all $g H \in G / H$.

Proposition 2.28. Let $H$ be a closed subgroup of $G$ and let $Y$ be an $H$-variety. Suppose that $B$ is a Borel subgroup of $G$ such that $\operatorname{dim} B \cap H$ is minimal (among all subgroups of the form $B^{\prime} \cap H$ for $B^{\prime}$ ranging over $\left.\mathscr{B}\right)$. Then we have

$$
\kappa_{G}\left(G *_{H} Y\right)=\kappa_{G}(G / H)+\kappa_{B \cap H}(Y) .
$$

Proof. We apply Theorem 2.8 to the morphism $\varphi: G *_{H} Y \rightarrow G / H$. Thus, for a Borel subgroup $B$ of $G$ and $g * y \in \Gamma_{G *_{H} Y}(B)$, we have that

$$
\kappa_{G}\left(G *_{H} Y\right)=\kappa_{G}(G / H)+\kappa_{K}(Z),
$$

where $Z$ is an irreducible component of $\varphi^{-1}(\varphi(g * y))$ passing through $g * y$ and $K=C_{B}(g H)^{\circ}$. Note that $C_{B}(g H)=B \cap g H^{-1}$. So, since $g * y \in \Gamma_{G * H} Y(B)$, the dimension of

$$
g^{-1} C_{B}(g H) g=g^{-1} B g \cap H
$$

is minimal. Now, as $G *_{H} Y$ is a fibre bundle, for $x \in G$ we have

$$
Y_{x}:=\varphi^{-1}(\varphi(x * y)) \cong Y .
$$

Define a morphism

$$
\phi: Y_{x} \rightarrow Y, \quad \phi(g * y)=x^{-1} g \cdot y .
$$

Clearly, $x h x^{-1} \in B \cap x H x^{-1}$ acts on $g * y \in Y_{x}$ as $x h x^{-1} \cdot(g * y)=x h x^{-1} g * y$. Since $g=x h^{\prime}$ for some $h^{\prime} \in H$, we have $x h x^{-1} \cdot(g * y)=x h h^{\prime} * y$. So $\phi\left(x h h^{\prime} * y\right)=h h^{\prime} \cdot y$. Thus, if we define an action of $B \cap x H x^{-1}$ on $Y$ by $x h x^{-1} \cdot y=h \cdot y$, the morphism $\phi: Y_{x} \rightarrow Y$ becomes a $\left(B \cap x H x^{-1}\right)$-equivariant isomorphism. It follows that $\kappa_{B \cap x H x^{-1}}\left(Y_{x}\right)=\kappa_{B \cap x H x^{-1}}(Y)$. Since $x^{-1}\left(B \cap x H x^{-1}\right) x=x^{-1} B x \cap H$, we finally get

$$
\kappa_{B \cap x H x^{-1}}(Y)=\kappa_{x^{-1} B x \cap H}(Y) .
$$

Next we need a technical lemma.

Lemma 2.29. Let $P$ be a parabolic subgroup of $G$. Then for $B$ ranging over $\mathscr{B}$, the intersection $B \cap P$ is minimal if and only if $B \cap P$ is a Borel subgroup of a Levi subgroup of $P$.

Proof. We may choose a Borel subgroup $B$ of $G$ so that $B P$ is open dense in $G$ (compare the proof of Lemma 2.11). Then the $P$-orbit of the base point in $G / B \cong \mathscr{B}$ is open dense in $\mathscr{B}$. Consequently, the stabilizer of this base point in $P$, $P \cap B$, is minimal among all the isotropy subgroups $P \cap B^{\prime}$ for $B^{\prime}$ in $\mathscr{B}$. Clearly, $B$ is opposite to a Borel subgroup of $G$ contained in $P$. Thanks to [Borel 1991, 
Corollary 14.13], $P \cap B$ contains a maximal torus $T$ of $G$. Let $L$ be the unique Levi subgroup of $P$ containing $T$. Then [Carter 1985, Theorem 2.8.7] implies that

$$
P \cap B=T\left(R_{u}(B) \cap L\right) .
$$

Clearly, $T\left(R_{u}(B) \cap L\right)$ is solvable and thus lies in a Borel subgroup of $L$. A simple dimension counting argument, using Theorem 2.7 applied to the multiplication map $B \times P \rightarrow B P$ and the fact that $\operatorname{dim} B P=\operatorname{dim} G$, shows that $P \cap B$ is a Borel subgroup of $L$.

Reversing the argument in the previous paragraph shows that if $P \cap B$ is a Borel subgroup of $L$, then $B P$ is dense in $G$ and thus $P \cap B$ is minimal again in the sense of the statement.

Next we consider a special case of Proposition 2.28.

Lemma 2.30. Let $P$ be a parabolic subgroup of $G$ and let $Y$ be a $P$-variety. Then

$$
\kappa_{G}\left(G *_{P} Y\right)=\kappa_{L}(Y),
$$

where $L$ is a Levi subgroup of $P$.

Proof. Proposition 2.28 implies that

$$
\kappa_{G}\left(G *_{P} Y\right)=\kappa_{G}(G / P)+\kappa_{B \cap P}(Y),
$$

where $\operatorname{dim} B \cap P$ is minimal. Lemmas 2.11 and 2.29 imply that $\kappa_{G}(G / P)=0$ and $B \cap P$ is a Borel subgroup of a Levi subgroup of $P$. The result follows.

Let $e \in \mathcal{N}$ be a nonzero nilpotent element, $\lambda \in \Omega_{G}^{a}(e)$ be an associated cocharacter of $e$ and $\mathfrak{g}=\bigoplus_{i \in \mathbb{Z}} \mathfrak{g}(i)$ be the grading of $\mathfrak{g}$ induced by $\lambda$. Also let $P$ be the destabilizing parabolic subgroup of $G$ defined by $e$ (see Section 2.5). In particular, we have Lie $P=\mathfrak{g}_{\geqslant 0}$ (see Section 2.6).

Lemma 2.31. Let $e \in \mathcal{N}$. Then

$$
G \cdot \mathfrak{g}_{\geqslant 2}=\overline{G \cdot e} .
$$

In particular, $\operatorname{dim} G \cdot \mathfrak{g}_{\geqslant 2}=\operatorname{dim} G \cdot e$.

Proof. Since $\mathfrak{g}_{\geqslant 2}$ is $P$-stable, $G \cdot \mathfrak{g}_{\geqslant 2}$ is closed [Humphreys 1995, Proposition 0.15]. Thus, since $e \in \mathfrak{g}(2) \subseteq \mathfrak{g} \geqslant 2$, we have

$$
\overline{G \cdot e} \subseteq G \cdot \mathfrak{g} \geqslant 2 .
$$

By Proposition $2.20, \overline{P \cdot e}=\mathfrak{g}_{\geqslant 2}$. Since $\overline{P \cdot e} \subseteq \overline{G \cdot e}$, we thus have $\mathfrak{g}_{\geqslant 2} \subseteq \overline{G \cdot e}$. Finally, as $\overline{G \cdot e}$ is $G$-stable,

$$
G \cdot \mathfrak{g} \geqslant 2 \subseteq \overline{G \cdot e}
$$


Theorem 2.32. Let $e \in \mathcal{N}$. Then

$$
\kappa_{G}(G \cdot e)=\kappa_{L}\left(\mathfrak{g}_{\geqslant 2}\right),
$$

where L is a Levi subgroup of $P$.

Proof. We have $\kappa_{G}(G \cdot e)=\kappa_{G}\left(G / C_{G}(e)\right)=\kappa_{G}\left(G / C_{P}(e)\right)$, thanks to (2-2) and the fact that $G_{G}(e)=C_{P}(e)$. Moreover, since $G *_{P} P / C_{P}(e) \cong G / C_{P}(e)$, it follows from Lemma 2.30 that $\kappa_{G}\left(G / C_{P}(e)\right)=\kappa_{L}\left(P / C_{P}(e)\right)$. Finally, thanks to Proposition 2.20 and (2-2), we obtain $\kappa_{L}\left(P / C_{P}(e)\right)=\kappa_{L}\left(\mathfrak{g}_{\geqslant 2}\right)$. The result follows.

Remark 2.33. For char $k=0$, Theorem 2.32 was proved by Panyushev [1999, Theorem 4.2.2].

Remark 2.34. Thanks to Theorem 2.32, in order to determine whether a nilpotent orbit is spherical, it suffices to show that a Borel subgroup of a Levi subgroup of $P$ acts on $\mathfrak{g}_{\geqslant 2}$ with a dense orbit. In our classification we pursue this approach.

2.8. Borel subgroups of Levi subgroups acting on unipotent radicals. Let $e \in \mathfrak{g}$ be a nonzero nilpotent element and let $\lambda \in \Omega_{G}^{a}(e)$ be an associated cocharacter for $e$. Let $P=P_{\lambda}$ be the destabilizing parabolic subgroup defined by $e$. We denote the Levi subgroup $C_{G}(\lambda)$ of $P$ by $L$. Our next result is taken from [Jantzen 2004, $\S 3]$. We only consider the case when $G$ is simple, the extension to the case when $G$ is reductive is straightforward.

Proposition 2.35. Let $G$ be a simple classical algebraic group and $0 \neq e \in \mathfrak{g}$ be nilpotent with corresponding partition $\pi_{e}=\left[1^{r_{1}}, 2^{r_{2}}, 3^{r_{3}}, \ldots\right]$. Let $a_{i}, b_{i}, s, t \in \mathbb{Z} \geqslant 0$ such that

$$
a_{i}+1=\sum_{j \geqslant i} r_{2 j+1}, b_{i}+1=\sum_{j \geqslant i} r_{2 j}, 2 s=\sum_{j \geqslant 0} r_{2 j+1}, \text { and } 2 t+1=\sum_{j \geqslant 0} r_{2 j+1} .
$$

Then the structure of $D L$ is as follows.

(i) If $G$ is of type $A_{n}$, then $\mathscr{D} L$ is of type $\prod_{i \geqslant 0} A_{a_{i}} \times \prod_{i \geqslant 1} A_{b_{i}}$.

(ii) If $G$ is of type $B_{n}$, then $\mathscr{D} L$ is of type $\prod_{i \geqslant 1} A_{a_{i}} \times \prod_{i \geqslant 1} A_{b_{i}} \times B_{t}$.

(iii) If $G$ is of type $C_{n}$, then $\mathscr{D} L$ is of type $\prod_{i \geqslant 1} A_{a_{i}} \times \prod_{i \geqslant 1} A_{b_{i}} \times C_{s}$.

(iv) If $G$ is of type $D_{n}$, then $\mathscr{D} L$ is of type $\prod_{i \geqslant 1} A_{a_{i}} \times \prod_{i \geqslant 1} A_{b_{i}} \times D_{s}$.

We use the conventions that

$$
A_{0}=B_{0}=C_{0}=D_{0}=\{1\}, \quad D_{1} \cong k^{*}, \quad \text { and } \quad D_{2}=A_{1} \times A_{1} .
$$

In order to describe the Levi subgroups $C_{G}(\lambda)$ for the exceptional groups we need to know more about associated cocharacters. Let $T$ be a maximal torus of $G$ such that $\lambda\left(k^{*}\right) \leqslant T$. Now let $G_{\mathbb{C}}$ be the simple, simply connected group over $\mathbb{C}$ with the same root system as $G$. Let $\mathfrak{g}_{\mathbb{C}}$ be the Lie algebra of $G_{\mathbb{C}}$. For a nilpotent 
element $e \in \mathfrak{g}_{\mathbb{C}}$ we can find an $\mathfrak{s l}_{2}$-triple containing $e$. Let $h \in \mathfrak{g}_{\mathbb{C}}$ be the semisimple element of this $\mathfrak{s l}_{2}$-triple. Note that $h$ is the image of 1 under the differential of $\lambda_{\mathbb{C}} \in G_{\mathbb{C}}$ (corresponding to $\lambda$ ) at 1 . Then there exists a set of simple roots $\Pi$ of $\Psi$ such that $\alpha(h) \geqslant 0$ for all $\alpha \in \Psi^{+}$and $\alpha(h)=m_{\alpha} \in\{0,1,2\}$ for all $\alpha \in \Pi$ [Carter $1985, \S 5.6]$. For each simple root $\alpha \in \Pi$ we attach the numerical label $m_{\alpha}$ to the corresponding node of the Dynkin diagram. The resulting labels form the weighted Dynkin diagram $\Delta(e)$ of $e$. We denote the set of weighted Dynkin diagrams of $G$ by $\mathscr{D}(\Pi)$. For $e, e^{\prime} \in \mathfrak{g}_{\mathbb{C}}$ nilpotent, we have that $\Delta(e)=\Delta\left(e^{\prime}\right)$ if and only if $e$ and $e^{\prime}$ are in the same $G_{\mathbb{C}}$-orbit.

In order to determine the weighted Dynkin diagram of a given nilpotent orbit we refer to the method outlined in [Carter 1985, §13] for the classical groups, and to the tables in the place cited for the exceptional groups.

We return to the case when the characteristic of $k$ is $\operatorname{good}$ for $G$. In this case the classification of the nilpotent orbits does not depend on the field $k$ [Carter 1985, $\S 5.11$ ]. Recently, Premet [2003] gave a proof of this fact for the unipotent classes of $G$ which is free from case-by-case considerations. This applies in our case, since the classification of the unipotent conjugacy classes in $G$ and of the nilpotent orbits in $\mathcal{N}$ is the same in good characteristic [Carter 1985, §9 and §11]. First assume that $G$ is simply connected and that $G$ admits a finite-dimensional rational representation such that the trace form on $\mathfrak{g}$ is nondegenerate; see [Premet 2003, $\S 2.3]$ for the motivation of these assumptions. Under these assumptions, given $\Delta \in \mathscr{D}(\Pi)$, there exists a cocharacter $\lambda=\lambda_{\Delta}$ of $G$ which is associated to $e$, where $e$ lies in the dense $L$-orbit in $\mathfrak{g}(2, \lambda)$, for $L=C_{G}(\lambda)$, such that

$$
\operatorname{Ad}(\lambda(t))\left(e_{ \pm \alpha}\right)=t^{ \pm m_{\alpha}} e_{ \pm \alpha} \quad \text { and } \quad \operatorname{Ad}(\lambda(t))(x)=x
$$

for all $\alpha \in \Pi, e_{ \pm \alpha} \in \mathfrak{g}_{ \pm \alpha}, x \in \mathfrak{t}$ and $t \in k^{*}$ [Premet 2003, §2.4]. We extend this action linearly to all of $\mathfrak{g}$. Now return to the general simple case. Let $\widehat{G}$ be the simple, simply connected group with the same root datum as $G$. Then there exists a surjective central isogeny

$$
\pi: \widehat{G} \rightarrow G
$$

(see [Carter 1985, §1.11]). Also, an associated cocharacter for $e=d \pi(\widehat{e})$ in $\mathfrak{g}$ is of the form $\pi \circ \widehat{\lambda}$, where $\widehat{\lambda}$ is a cocharacter of $\widehat{G}$ that is associated to $\widehat{e}$ in $\widehat{\mathfrak{g}}$. This implies that (2-3) holds for an arbitrary simple algebraic group, when the characteristic of $k$ is good for $G$.

After these deliberations we can use the tables in [Carter 1985, §13] to determine the structure of the Levi subgroups $C_{G}(\lambda)$ for the exceptional groups. Recall that Lie $C_{G}(\lambda)=\mathfrak{g}(0)$ and $\mathfrak{g}(0)$ is the sum of the root spaces $\mathfrak{g}_{\alpha}$, where $\alpha \in \Psi$ with $\langle\alpha, \lambda\rangle=0$. Let $\Pi_{0}=\left\{\alpha \in \Pi \mid m_{\alpha}=0\right\}$, the set of nodes $\alpha$ of the corresponding 
weighted Dynkin diagram with label $m_{\alpha}=0$. Then

$$
C_{G}(\lambda)=\left\langle T, U_{ \pm \alpha} \mid \alpha \in \Pi_{0}\right\rangle .
$$

It is straightforward to determine the height of a nilpotent orbit from its associated weighted Dynkin diagram. Let $\tilde{\alpha}=\sum_{\alpha \in \Pi} c_{\alpha} \alpha$ be the highest root of $\Psi$. For each simple root $\alpha \in \Pi$ we have $\mathfrak{g}_{\alpha} \subseteq \mathfrak{g}\left(m_{\alpha}\right)$ where $m_{\alpha}$ is the corresponding numerical label on the weighted Dynkin diagram, by (2-3).

Lemma 2.36. Let $\tilde{\alpha}$ be the highest root of $\Psi$ and set $d=h t(e)$. Then $\mathfrak{g}_{\tilde{\alpha}} \subseteq \mathfrak{g}(d)$.

Proof. Clearly, we have $\mathfrak{g}_{\tilde{\alpha}} \subseteq \mathfrak{g}(i)$ for some $i \geqslant 0$. The lemma is immediate, because if

$$
\tilde{\alpha}=\sum_{\alpha \in \Pi} c_{\alpha} \alpha, \quad \text { and } \quad \beta=\sum_{\alpha \in \Pi} d_{\alpha} \alpha
$$

is any other root of $\Psi$, then $c_{\alpha} \geqslant d_{\alpha}$ for all $\alpha \in \Pi$.

Lemma 2.36 readily implies

$$
\operatorname{ht}(e)=\sum_{\alpha \in \Pi} m_{\alpha} c_{\alpha} .
$$

The identity (2-4) is also observed in [Panyushev 1994, §2.1].

For the remainder of this section we assume that $G$ is simple. The generalization of each of the subsequent results to the case when $G$ is reductive is straightforward.

For $P$ a parabolic subgroup of $G$ we set $\mathfrak{p}_{u}=$ Lie $R_{u}(P)$.

Proposition 2.37. Let $P=L R_{u}(P)$ be an arbitrary parabolic subgroup of $G$, where $L$ is a Levi subgroup of $P$. Then

$$
\kappa_{G}(G / L)=\kappa_{L}(P / L)=\kappa_{L}\left(R_{u}(P)\right)=\kappa_{L}\left(\mathfrak{p}_{u}\right) .
$$

Proof. Thanks to Lemma 2.30, we have $\kappa_{G}(G / L)=\kappa_{G}\left(G *_{P} P / L\right)=\kappa_{L}(P / L)$.

If we write $P=R_{u}(P) L$, then the bijection $P / L=R_{u}(P) L / L \cong R_{u}(P)$ gives a canonical $L$-equivariant isomorphism $\phi: P / L \rightarrow R_{u}(P)$ defined by $\phi(x L)=y$, where $x=y z$ with $y \in R_{u}(P)$ and $z \in L$. Thus, we have $\kappa_{L}(P / L)=\kappa_{L}\left(R_{u}(P)\right)$.

A Springer isomorphism between the unipotent variety of $G$ and $\mathcal{N}$ restricts to an $L$-equivariant isomorphism $R_{u}(P) \rightarrow \mathfrak{p}_{u}$ [Goodwin 2005b, Corollary 1.4], so that $\kappa_{L}\left(R_{u}(P)\right)=\kappa_{L}\left(\mathfrak{p}_{u}\right)$.

Remarks 2.38. (i) While the first two equalities of Proposition 2.37 hold in arbitrary characteristic, the third equality requires the characteristic of the underlying field to be zero or a good prime for $G$; this assumption is required for the existence of a Springer isomorphism (see [Goodwin 2005b, Corollary 1.4]).

(ii) [Brundan 1998, Lemma 4.2] states that there is a dense $L$-orbit on $G / B$ if and only if there is a dense $B_{L}$-orbit on $R_{u}(P)$, where $B_{L}$ is a Borel subgroup of $L$. 
Notice that there is a dense $L$-orbit on $G / B$ if and only if there is a dense $B$-orbit on $G / L$. In other words, $\kappa_{G}(G / L)=0$ if and only if $\kappa_{L}\left(R_{u}(P)\right)=0$. Thus, Proposition 2.37 generalizes [Brundan 1998, Lemma 4.2].

By Proposition 2.37, the problem of determining $\kappa_{L}\left(R_{u}(P)\right)$ is equivalent to the problem of determining $\kappa_{G}(G / L)$. In particular, a Borel subgroup of $L$ acts on $R_{u}(P)$ with a dense orbit if and only if $L$ is a spherical subgroup of $G$. In fact, the latter have been classified. In characteristic zero this result was proved by M. Krämer [1979] and extended to arbitrary characteristic by J. Brundan [1998, Theorem 4.1].

Theorem 2.39. Let $L$ be a proper Levi subgroup of a simple group $G$. Then $L$ is spherical in $G$ if and only if $(G, \mathscr{D} L)$ is one of

$$
\begin{aligned}
& \left(A_{n}, A_{i-1} A_{n-i}\right),\left(B_{n}, B_{n-1}\right),\left(B_{n}, A_{n-1}\right),\left(C_{n}, C_{n-1}\right), \\
& \left(C_{n}, A_{n-1}\right),\left(D_{n}, D_{n-1}\right),\left(D_{n}, A_{n-1}\right),\left(E_{6}, D_{5}\right),\left(E_{7}, E_{6}\right) .
\end{aligned}
$$

We also recall the classification of the parabolic subgroups of $G$ with an abelian unipotent radical (see [Richardson et al. 1992, Lemma 2.2]).

Lemma 2.40. Let $G$ be a simple algebraic group and $P$ be a parabolic subgroup of $G$. Then $R_{u}(P)$ is abelian if and only if $P$ is a maximal parabolic subgroup of $G$ which is conjugate to the standard parabolic subgroup $P_{I}$ of $G$, where $I=\Pi \backslash\{\alpha\}$ and $\alpha$ occurs in the highest root $\tilde{\alpha}$ with coefficient 1.

Let $\Pi=\left\{\alpha_{1}, \alpha_{2}, \ldots, \alpha_{n}\right\}$ be a set of simple roots of the root system $\Psi$ of $G$. Using Lemma 2.40, we can readily determine the standard parabolic subgroups $P_{I}$ of $G$ with an abelian unipotent radical. For $G$ simple we gather this information in Table 1 along with the structure of the corresponding standard Levi subgroup $L_{I}$ of $P_{I}$. Set $P_{\alpha_{i}^{\prime}}=P_{\Pi \backslash\left\{\alpha_{i}\right\}}$. Here the simple roots are labelled as in [Bourbaki 1968, Planches I-IX].

Note that if $G$ is of type $E_{8}, F_{4}$ or $G_{2}$, then $G$ does not admit a parabolic subgroup with an abelian unipotent radical. Also compare the list of pairs $(G, \mathscr{D} L)$ from Table 1 with the list in Theorem 2.39.

\begin{tabular}{c|c|c} 
Type of $G$ & $P_{I}$ & Type of $\mathscr{D} L_{I}$ \\
\hline$A_{n}$ & $P_{\alpha_{i}^{\prime}}$ for $1 \leqslant i \leqslant n$ & $A_{i-1} A_{n-i}$ \\
$B_{n}$ & $P_{\alpha_{1}^{\prime}}$ & $B_{n-1}$ \\
$C_{n}$ & $P_{\alpha_{n}^{\prime}}$ & $A_{n-1}$ \\
$D_{n}$ & $P_{\alpha_{1}^{\prime}}, P_{\alpha_{n-1}^{\prime}}$ and $P_{\alpha_{n}^{\prime}}$ & $D_{n-1}$ or $A_{n-1}$ \\
$E_{6}$ & $P_{\alpha_{1}^{\prime}}$ and $P_{\alpha_{6}^{\prime}}$ & $D_{5}$ \\
$E_{7}$ & $P_{\alpha_{7}^{\prime}}$ & $E_{6}$
\end{tabular}

Table 1. Parabolic subgroups with abelian unipotent radical. 
Our next result is immediate from [Brundan 1998, Theorem 4.1, Lemma 4.2]. Proposition 2.41. If $P=L R_{u}(P)$ is a parabolic subgroup of $G$ with $R_{u}(P)$ abelian, then $\kappa_{L}\left(R_{u}(P)\right)=0$.

Proof. If $R_{u}(P)$ is abelian, then using Table 1 we see that all the possible pairs $(G, \mathscr{D} L)$ appear in the list of spherical Levi subgroups given in Theorem 2.39, that is, $\kappa_{G}(G / L)=0$. Proposition 2.37 then implies that $\kappa_{L}\left(R_{u}(P)\right)=0$.

Corollary 2.42. If $P$ is a parabolic subgroup of $G$ with $R_{u}(P)$ abelian, then $\kappa_{L}\left(\mathfrak{p}_{u}\right)=0$.

Let $\Psi$ be the root system of $G$ and let $\Pi \subseteq \Psi$ be a set of simple roots of $\Psi$. Let $P=P_{I}(I \subseteq \Pi)$ be a standard parabolic subgroup of $G$. Let $\Psi_{I}$ be the root system of the standard Levi subgroup $L_{I}$, that is, $\Psi_{I}$ is spanned by $I$. Define $\Psi_{I}^{+}=\Psi_{I} \cap \Psi^{+}$. For any root $\alpha \in \Psi$ we can uniquely write $\alpha=\alpha_{I}+\alpha_{I^{\prime}}$ where

$$
\alpha_{I}=\sum_{\beta \in I} c_{\beta} \beta, \quad \text { and } \quad \alpha_{I^{\prime}}=\sum_{\beta \in \Pi \backslash I} d_{\beta} \beta .
$$

We define the level of $\alpha$ (relative to $P$ or relative to $I$ ) to be

$$
\operatorname{lv}(\alpha):=\sum_{\beta \in \Pi \backslash I} d_{\beta}
$$

(see [Azad et al. 1990]). Let $d$ be the maximal level of any root in $\Psi$. If $2 i>d$, then

$$
A_{i}:=\prod_{\operatorname{lv}(\alpha)=i} U_{\alpha}
$$

is an abelian unipotent subgroup of $G$. Note $A_{d}$ is the centre of $R_{u}(P)$. Since $L$ normalizes each $A_{i}$, we can consider $\kappa_{L}\left(A_{i}\right)$.

Proposition 2.43. If $P$ is a parabolic subgroup of $G$ and $2 i>d$, then $\kappa_{L}\left(A_{i}\right)=0$. Proof. We maintain the setup from the previous paragraph. Set $A_{i}$ as in (2-5) and

$$
A_{i}^{-}=\prod_{\operatorname{lv}(\alpha)=-i} U_{\alpha}
$$

Let $H$ be the subgroup of $G$ generated by $A_{i}, A_{i}^{-}$, and $L$. Then $H$ is reductive, with root system

$$
\Psi_{I} \cup\{\alpha \in \Psi \mid \operatorname{lv}(\alpha)= \pm i\},
$$

and $L A_{i}$ is a parabolic subgroup of $H$. Since $A_{i}$ is abelian, we can invoke Proposition 2.41 to deduce that $\kappa_{L}\left(A_{i}\right)=0$. 
There is a natural Lie algebra analogue of Proposition 2.43: Maintaining the setup from above, for $2 i>d$, we see that

$$
\mathfrak{a}_{i}:=\bigoplus_{\operatorname{lv}(\alpha)=i} \mathfrak{g}_{\alpha}
$$

is an abelian subalgebra of $\mathfrak{g}$. Since Lie $U_{\alpha}=\mathfrak{g}_{\alpha}$ for all $\alpha \in \Psi$, we have Lie $A_{i}=\mathfrak{a}_{i}$. Thanks to [Goodwin 2005b, Corollary 1.4], we obtain the following consequence of Proposition 2.43.

Corollary 2.44. If $P$ is a parabolic subgroup of $G$ and $2 i>d$, then $\kappa_{L}\left(\mathfrak{a}_{i}\right)=0$.

Remarks 2.45. (i) Corollary 2.44 was first proved, for a field of characteristic zero, in [Panyushev 1994, Proposition 3.2]; the proof there is somewhat different from ours.

(ii) Propositions 2.41 and 2.43 suggest that if $A$ is an abelian subgroup of $R_{u}(P)$ which is normal in $P$, then $\kappa_{L}(A)=0$. It is indeed the case that $P$ acts on $A$ with a dense orbit [Röhrle 1998, Theorem 1.1]. However, this is not the case when we consider instead the action of a Borel subgroup of a Levi subgroup of $P$ on $A$. For example, it follows from [Röhrle 1998, Table 1] that if $G$ is of type $A_{n}$, then the dimension of a maximal normal abelian subgroup $A$ of a Borel subgroup $B$ of $G$ is $i(n+1-i)$, where $1 \leqslant i \leqslant n$. Clearly, for $1 \neq i \neq n$ we have $\operatorname{dim} A>\operatorname{rk} G$. Thus, a maximal torus of $B$ cannot act on $A$ with a dense orbit. Using [Röhrle 1998, Table 1], it is easy to construct further examples.

\section{The classification of the spherical nilpotent orbits}

3.1. Height two nilpotent orbits. We will now show that height two nilpotent orbits are spherical. Let $e \in \mathfrak{g}$ be nilpotent and let $\lambda \in \Omega_{G}^{a}(e)$ be an associated cocharacter of $G$. Define the following subalgebra of $\mathfrak{g}$ :

$$
\mathfrak{g}_{E}:=\bigoplus_{i \in \mathbb{Z}} \mathfrak{g}(2 i)
$$

Proposition 3.1. Let $e \in \mathcal{N}, \lambda \in \Omega_{G}^{a}(e)$, and let $\mathfrak{g}_{E}$ be the subalgebra of $\mathfrak{g}$ defined in (3-1).

(i) There exists a connected reductive subgroup $G_{E}$ of $G$ such that Lie $G_{E}=\mathfrak{g}_{E}$.

(ii) There exists a parabolic subgroup $Q$ of $G_{E}$ such that

$$
\text { Lie } Q=\bigoplus_{i \geqslant 0} \mathfrak{g}(2 i) \text {. }
$$


Moreover, $C_{G}(\lambda)$ is a Levi subgroup of $Q$ and

$$
\text { Lie } R_{u}(Q)=\bigoplus_{i \geqslant 1} \mathfrak{g}(2 i)
$$

Proof. Fix a maximal torus $T$ of $G$ such that $\lambda\left(k^{*}\right) \leqslant T$. Set

$$
\Phi=\{\alpha \in \Psi \mid\langle\alpha, \lambda\rangle \in 2 \mathbb{Z}\} .
$$

So $\mathfrak{g}_{E}=\bigoplus_{\alpha \in \Phi} \mathfrak{g}_{\alpha}$.

Then $\Phi$ is a semisimple subsystem of $\Psi$. The subgroup $G_{E}$ generated by $T$ and all the one-dimensional root subgroups $U_{\alpha}$ with $\alpha \in \Phi$ is reductive and has Lie algebra $\mathfrak{g}_{E}$.

Let $Q=P \cap G_{E}$, where $P=P_{\lambda}$. Since $\lambda\left(k^{*}\right) \leqslant T \leqslant G_{E}$, we see that $Q$ is a parabolic subgroup of $G_{E}$; see the remarks preceding Theorem 2.14. Since Lie $C_{G}(\lambda)=\mathfrak{g}(0)$, we have $C_{G}(\lambda) \leqslant Q$ and so $C_{G}(\lambda)$ is a Levi subgroup of $Q$. The remaining claims follow from the fact that Lie $P=\mathfrak{g} \geqslant 0$, the parabolic subgroup $P$ has Levi decomposition $P=C_{G}(\lambda) R_{u}(P)$ and Lie $R_{u}(P)=\mathfrak{g}_{>0}$.

The following discussion and Lemma 3.2 allow us to reduce the determination of the spherical nilpotent orbits to the case when $G$ is simple. Since the centre of $G$ acts trivially on $\mathfrak{g}$, we may assume that $G$ is semisimple. Let $\widetilde{G}$ be semisimple of adjoint type and $\pi: G \rightarrow \widetilde{G}$ be the corresponding isogeny. Let $e \in \mathfrak{g}$ be nilpotent and let $\tilde{e}=d \pi_{1}(e)$. Consider the restriction of $d \pi_{1}$ to the nilpotent variety of $\mathfrak{g}$. Then $d \pi_{1}: \mathcal{N} \rightarrow \widetilde{\mathcal{N}}$ is a dominant $G$-equivariant morphism, where $\widetilde{\mathcal{N}}$ is the nilpotent variety of Lie $\widetilde{G}$ and $G$ acts on $\widetilde{\mathcal{N}}$ via $\widetilde{A d} \circ \pi$. It then follows from Theorem 2.8 that $\kappa_{G}(G \cdot e)=\kappa_{\widetilde{G}}(\widetilde{G} \cdot \tilde{e})$. We therefore may assume that $G$ is semisimple of adjoint type.

Lemma 3.2. Let $G$ be semisimple of adjoint type. Then $G$ is a direct product of simple groups $G=G_{1} G_{2} \cdots G_{r}$. If $e \in \mathfrak{g}$ is nilpotent, then $e=e_{1}+e_{2}+\cdots+e_{r}$ for $e_{i}$ nilpotent in $\mathfrak{g}_{i}=\operatorname{Lie} G_{i}$ and $\kappa_{G}(G \cdot e)=\sum_{i=1}^{r} \kappa_{G_{i}}\left(G_{i} \cdot e_{i}\right)$.

Proof. Since $G$ is semisimple of adjoint type, so that $G$ is the direct product $G=G_{1} G_{2} \cdots G_{r}$ of simple groups $G_{i}$, we have Lie $G=\oplus$ Lie $G_{i}$. Let $e \in \mathfrak{g}$ be nilpotent. Clearly, any element $x \in C_{G}(e)$ is of the form $x=x_{1} x_{2} \cdots x_{r}$ where $x_{i} \in G_{i}$ and we also have that $e=e_{1}+e_{2}+\cdots+e_{r}$, where $e_{i} \in \mathfrak{g}_{i}$ and each $e_{i}$ must be nilpotent. We know that $\operatorname{Ad}(x)(e)=e$ so

$$
\operatorname{Ad}\left(x_{1}\right) \operatorname{Ad}\left(x_{2}\right) \cdots \operatorname{Ad}\left(x_{r}\right)\left(e_{1}+e_{2}+\cdots+e_{r}\right)=e_{1}+e_{2}+\cdots+e_{r} .
$$

For $i \neq j$ we have $\operatorname{Ad}\left(x_{i}\right)\left(e_{j}\right)=e_{j}$, so $\operatorname{Ad}(x)\left(e_{i}\right)=\operatorname{Ad}\left(x_{i}\right)\left(e_{i}\right)$. Therefore, as $\operatorname{Ad}\left(x_{i}\right)$ stabilizes $\mathfrak{g}_{i}$, we have $\operatorname{Ad}\left(x_{i}\right)\left(e_{i}\right)=e_{i}$. Thus, we obtain the following decomposition $C_{G}(e)=C_{G_{1}}\left(e_{1}\right) C_{G_{2}}\left(e_{2}\right) \cdots C_{G_{r}}\left(e_{r}\right)$. For $B$ a Borel subgroup of $G$ we have $B=B_{1} B_{2} \cdots B_{r}$, where each $B_{i}$ is a Borel subgroup of $G_{i}$ and $C_{B}(e)=$ 
$C_{B_{1}}\left(e_{1}\right) C_{B_{2}}\left(e_{2}\right) \cdots C_{B_{r}}\left(e_{r}\right)$. In particular, for $B \in \Gamma_{G}(e)$ we have that $\operatorname{dim} C_{B}(e)$ is minimal. This implies that $\operatorname{dim} C_{B_{i}}\left(e_{i}\right)$ is minimal for each $i$ and so $B_{i} \in \Gamma_{G_{i}}\left(e_{i}\right)$. Therefore, we have

$$
\begin{aligned}
\kappa_{G}(G \cdot e) & =\operatorname{dim} G-\operatorname{dim} C_{G}(e)-\operatorname{dim} B+\operatorname{dim} C_{B}(e) \\
& =\sum_{i=1}^{r} \operatorname{dim} G_{i}-\sum_{i=1}^{r} \operatorname{dim} C_{G_{i}}\left(e_{i}\right)-\sum_{i=1}^{r} \operatorname{dim} B_{i}+\sum_{i=1}^{r} \operatorname{dim} C_{B_{i}}\left(e_{i}\right) \\
& =\sum_{i=1}^{r}\left(\operatorname{dim} G_{i}-\operatorname{dim} C_{G_{i}}\left(e_{i}\right)-\operatorname{dim} B_{i}+\operatorname{dim} C_{B_{i}}\left(e_{i}\right)\right) \\
& =\sum_{i=1}^{r} \kappa_{G_{i}}\left(G_{i} \cdot e_{i}\right) .
\end{aligned}
$$

Lemma 3.3. Let $G$ be a connected reductive algebraic group and $e \in \mathfrak{g}$ be nilpotent. If $\mathrm{ht}(e)=2$, then $e$ is spherical.

Proof. First we assume that $G$ is simple. Let $\lambda \in \Omega_{G}^{a}(e)$. Let $\mathfrak{g}_{E}$ be the Lie subalgebra of $\mathfrak{g}$ as defined in (3-1) and let $Q$ be the parabolic subgroup of $G_{E}$ as in Proposition 3.1(ii). Since ht $(e)=2$, we have $\mathfrak{g}_{E}=\mathfrak{g}(-2) \oplus \mathfrak{g}(0) \oplus \mathfrak{g}(2)$. Set $L=C_{G}(\lambda)$. Then $\kappa_{G}(G \cdot e)=\kappa_{L}(\mathfrak{g}(2))$, by Theorem 2.32. Also, by Proposition 3.1, Lie $R_{u}(Q)=\mathfrak{g}(2)$. Since $R_{u}(Q)$ is abelian, Corollary 2.42 implies that $\kappa_{L}(\mathfrak{g}(2))=0$.

Now suppose that $G$ is reductive. Let $\mathscr{D} G=G_{1} G_{2} \cdots G_{r}$ be a commuting product of simple groups. For $e \in \mathfrak{g}$ we have $e=e_{1}+e_{2}+\cdots+e_{r}$, where $e_{i} \in$ $\mathfrak{g}_{i}=$ Lie $G_{i}$ and each $e_{i}$ is nilpotent. Since ht $(e)=\max _{1 \leqslant i \leqslant r} \operatorname{ht}\left(e_{i}\right)$, we have $\operatorname{ht}\left(e_{i}\right) \leqslant \mathrm{ht}(e)=2$ for all $i$. Since $\kappa_{G}(G \cdot e)=\sum_{i=1}^{r} \kappa_{G_{i}}\left(G_{i} \cdot e_{i}\right)$, by Lemma 3.2, the result follows from the simple case just proved.

3.2. Even gradings. Suppose that the given nilpotent element $e \in \mathfrak{g}$ satisfies

$$
\operatorname{ht}(e) \geqslant 4 \text {. }
$$

Also assume that any $\lambda \in \Omega_{G}^{a}(e)$ induces an even grading on $\mathfrak{g}$, that is, $\mathfrak{g}(i, \lambda)=\{0\}$, whenever $i$ is odd. As usual we denote $\mathfrak{g}(i, \lambda)$ simply by $\mathfrak{g}(i)$.

Lemma 3.4. Let $e \in \mathcal{N}$ and $\lambda \in \Omega_{G}^{a}(e)$ be as above. Then $\mathfrak{g}_{\geqslant 2}$ is nonabelian.

Proof. Set ht $(e)=d$. For the highest root $\tilde{\alpha} \in \Psi^{+}$we have $\mathfrak{g}_{\tilde{\alpha}} \subseteq \mathfrak{g}(d)$. Write $\tilde{\alpha}=\alpha_{1}+\alpha_{2}+\cdots+\alpha_{r}$ as a sum of not necessarily distinct simple roots. The sequence of simple roots $\alpha_{1}, \alpha_{2}, \ldots, \alpha_{r}$ can be chosen so that $\alpha_{1}+\alpha_{2}+\cdots+\alpha_{s}$ is a root for all $1 \leqslant s \leqslant r$ [Humphreys 1972, Corollary 10.2.A]. Since the grading of $\mathfrak{g}$ induced by $\lambda$ is even, for all simple roots $\alpha \in \Pi$, we have $\mathfrak{g}_{\alpha} \subseteq \mathfrak{g}(i)$ with $i \in\{0,2\}$ (compare (2-3)). Since $d \geqslant 4$, for at least one $\alpha_{i}$ we must have $\mathfrak{g}_{\alpha_{i}} \subseteq \mathfrak{g}(2)$. Let $\alpha_{k}$ 
be the last simple root in the sequence $\alpha_{1}, \alpha_{2}, \ldots, \alpha_{r}$ with this property. Thus, for $\beta=\alpha_{1}+\alpha_{2}+\cdots+\alpha_{k-1}$ we have $\mathfrak{g}_{\beta} \in \mathfrak{g}(d-2) \subseteq \mathfrak{g} \geqslant 2$. Since char $k$ is good for $G$, we have $\left[\mathfrak{g}_{\beta}, \mathfrak{g}_{\alpha_{k}}\right]=\mathfrak{g}_{\beta^{\prime}}$ where $\beta^{\prime}=\beta+\alpha_{k}$. Therefore, $\mathfrak{g}_{\geqslant 2}$ is nonabelian.

Corollary 3.5. Let $P$ be the destabilizing parabolic subgroup of $G$ defined by $e \in$ $\mathcal{N}$. Then $R_{u}(P)$ is nonabelian.

Set $\mathfrak{p}_{u}=$ Lie $R_{u}(P)$. Because the grading of $\mathfrak{g}$ is even, $\mathfrak{g}_{\geqslant 2}=\mathfrak{p}_{u}$. Thus, by Proposition 2.37 and Theorem 2.32, we have $\kappa_{G}(G \cdot e)=\kappa_{G}(G / L)$, where $L=C_{G}(\lambda)$. Using the classification of the spherical Levi subgroups and the classification of the parabolic subgroups of $G$ with abelian unipotent radical, Theorem 2.39 and Lemma 2.40, we see that there are only two cases, for $G$ simple, when $R_{u}(P)$ is nonabelian and $L$ is spherical, namely when $G$ is of type $B_{n}$ and $\mathscr{D} L$ is of type $A_{n-1}$ and when $G$ is of type $C_{n}$ and $\mathscr{D} L$ is of type $C_{n-1}$.

Lemma 3.6. Let $G$ be of type $B_{n}$ or of type $C_{n}$. Let $e \in \mathcal{N}$ and $\lambda \in \Omega_{G}^{a}(e)$. Set $L=C_{G}(\lambda)$. If $\pi_{e}=\left[1^{r_{1}}, 2^{r_{2}}, \ldots\right]$ is the corresponding partition for $e$, then

$$
\operatorname{dim} Z(L)=\left|\left\{a_{i}, b_{i} \in \mathbb{Z} \geqslant 0 \mid a_{i}+1=\sum_{j \geqslant i} r_{2 j+1}, b_{i}+1=\sum_{j \geqslant i} r_{2 j}\right\}\right| .
$$

Proof. Since $L$ is reductive, $L=Z(L) \mathscr{D}$, and $Z(L) \cap \mathscr{D} L$ is finite, we have $\operatorname{dim} L=\operatorname{dim} Z(L)+\operatorname{dim} \mathscr{D} L$. The result follows from Proposition 2.35.

It is straightforward to deduce the following from Propositions 2.26 and 2.35.

Lemma 3.7. Let $e \in \mathcal{N}$ and $\lambda \in \Omega_{G}^{a}(e)$ with $\mathrm{ht}(e) \geqslant 4$. Set $L=C_{G}(\lambda)$. If $G$ is of type $B_{n}$, then $D L$ is not of type $A_{n-1}$ and if $G$ is of type $C_{n}$, then $\$ L$ is not of type $C_{n-1}$.

Lemma 3.8. Let $e \in \mathcal{N}$ and suppose that $\lambda \in \Omega_{G}^{a}(e)$ induces an even grading on $\mathfrak{g}$. If $\mathrm{ht}(e) \geqslant 4$, then $e$ is nonspherical.

Proof. First we observe that if $G$ is simple, then the statement follows from the facts that $R_{u}(P)$ is nonabelian (Corollary 3.5) and that $(G, \mathscr{D} L)$ is not one of the pairs $\left(B_{n}, A_{n-1}\right)$ or $\left(C_{n}, C_{n-1}\right)$ (Lemma 3.7). So by Theorem 2.39 and Lemma 2.40, we see that $L$ is a nonspherical subgroup. Therefore, by Proposition 2.37, $\kappa_{L}\left(\mathfrak{g}_{\geqslant 2}\right)>0$ and $e$ is nonspherical.

In case $G$ is reductive, we argue as in the proof of Lemma 3.3 and reduce to the simple case.

3.3. Nilpotent orbits of height at least four. Let $e \in \mathfrak{g}$ be nilpotent and let $\lambda \in$ $\Omega_{G}^{a}(e)$. Let $\mathfrak{g}_{E}$ be the subalgebra of $\mathfrak{g}$ as defined in (3-1). Also let $G_{E}$ be the connected reductive algebraic group such that Lie $G_{E}=\mathfrak{g}_{E}$ and $Q$ be the parabolic subgroup of $G_{E}$ as in Proposition 3.1(ii). 
Since $e \in \mathfrak{g}_{E}$ and $\lambda\left(k^{*}\right) \leqslant G_{E}$, it follows from [Fowler and Röhrle 2008, Theorem 1.1] that $\lambda$ is a cocharacter of $G_{E}$ which is associated to $e$, that is, $\lambda \in \Omega_{G_{E}}^{a}(e)$. Moreover, for $P=P_{\lambda}$, we have $Q=P \cap G_{E}$ is the destabilizing parabolic subgroup of $G_{E}$ defined by $e$.

Let ht $E_{E}(e)$ denote the height of $e \in \mathfrak{g}_{E}$. Now if ht $(e) \geqslant 4$ and ht $(e)$ is even, then $\mathrm{ht}_{E}(e)=\mathrm{ht}(e)$. The case when ht $(e) \geqslant 4$ and ht $(e)$ is odd is slightly more involved. First we need some preliminary results. A proof of the following can be found in [Panyushev 1999, Proposition 2.4].

Lemma 3.9. Suppose that $\operatorname{char} k=0$. If $e \in \mathcal{N}$ with $\mathrm{ht}(e)$ odd, then the weighted Dynkin diagram $\Delta(e)$ contains no " 2 " labels.

If $\Pi$ is a set of simple roots of $\Psi$ relative to a maximal torus $T$ which contains $\lambda\left(k^{*}\right)$, then for $\alpha \in \Pi$ we have

$$
\mathfrak{g}_{\alpha} \subseteq \mathfrak{g}(i),
$$

where $i \in\{0,1\}$. To see this recall (2-3): $\operatorname{Ad}(\lambda(t))\left(e_{\alpha}\right)=t^{m_{\alpha}} e_{\alpha}$, for $e_{\alpha} \in \mathfrak{g}_{\alpha}$ and $m_{\alpha}$ is the corresponding label of the weighted Dynkin diagram $\Delta(e)$ of $e$. Thus, by Lemma 3.9, we have $m_{\alpha} \in\{0,1\}$.

Lemma 3.10. If $\mathrm{ht}(e)=d$ is odd, then $\mathfrak{g}(d-1) \neq\{0\}$.

Proof. The result follows easily, arguing as in the proof of Lemma 3.4 and using (3-2).

Corollary 3.11. If $e \in \mathcal{N}$ with ht $(e)$ odd, then $\mathrm{ht}_{E}(e)=\mathrm{ht}(e)-1$.

In particular, we have the following conclusion.

Corollary 3.12. If $e \in \mathcal{N}$ with ht $(e) \geqslant 4$, then $\mathrm{ht}_{E}(e) \geqslant 4$.

Thus, by Lemma 3.8, Corollary 3.12, and the fact that

$$
\Omega_{G}^{a}(e) \cap Y\left(G_{E}\right)=\Omega_{G_{E}}^{a}(e)
$$

(see [Fowler and Röhrle 2008, Theorem 1.1]), we have $\kappa_{L}\left(\mathfrak{g}_{E, \geqslant 2}\right)>0$, where

$$
\mathfrak{g}_{E, \geqslant 2}=\bigoplus_{i \geqslant 1} \mathfrak{g}(2 i) \quad \text { and } \quad L=C_{G}(\lambda)=C_{G_{E}}(\lambda)
$$

Lemma 3.13. If a Borel subgroup $B_{L}$ of $L$ acts on $\mathfrak{g}_{\geqslant 2}$ with a dense orbit, then $B_{L}$ acts on $\mathfrak{g}_{E, \geqslant 2}$ with a dense orbit.

Proof. This follows readily from Theorem 2.9.

Combining Lemmas 3.8, 3.13 and Corollary 3.12, we get the main result of this section.

Proposition 3.14. Let $e \in \mathcal{N}$. If $\operatorname{ht}(e) \geqslant 4$, then e is nonspherical. 
3.4. Nilpotent orbits of height three. Let $e \in \mathcal{N}$ and let $\lambda \in \Omega_{G}^{a}(e)$. Let $P=P(e)$ be the destabilizing parabolic subgroup defined by $e$. Then $P=L R_{u}(P)$ for $L=$ $C_{G}(\lambda)$. Let $B_{L}$ be a Borel subgroup of $L$ so that $\lambda\left(k^{*}\right) \leqslant B_{L}$. Write $B_{L}=T U_{L}$ for a Levi decomposition of $B_{L}$, where $U_{L}=R_{u}\left(B_{L}\right)$ and $T$ is a maximal torus of $G$ containing $\lambda\left(k^{*}\right)$. Let $\mathfrak{b}_{L}=\operatorname{Lie} B_{L}, \mathfrak{n}=\operatorname{Lie} U_{L}$, and $\mathfrak{t}=\operatorname{Lie} T$.

Lemma 3.15. Let $e \in \mathfrak{g}$ be nilpotent and $\lambda$ be an associated cocharacter for $e$ in $\mathfrak{g}$. Then the following are equivalent.

(i) The nilpotent element e is spherical.

(ii) There exists $e^{\prime} \in \mathfrak{g}_{\geqslant 2}$ such that $\overline{\operatorname{Ad}\left(B_{L}\right)\left(e^{\prime}\right)}=\mathfrak{g}_{\geqslant 2}$.

(iii) There exists $e^{\prime} \in \mathfrak{g} \geqslant 2$ such that $\operatorname{dim} C_{B_{L}}\left(e^{\prime}\right)=\operatorname{dim} B_{L}-\operatorname{dim} \mathfrak{g} \geqslant 2$.

Proof. Thanks to Theorem 2.32, $\kappa_{G}(G \cdot e)=\kappa_{L}\left(\mathfrak{g}_{\geqslant 2}\right)$. Thus (i) and (ii) are equivalent. The equivalence between (ii) and (iii) is clear.

Recall from Section 2.1 the definition of the support of a nilpotent element in $\mathfrak{u}$.

Lemma 3.16. Let $e \in \mathfrak{g} \geqslant 2$. If $\operatorname{supp}(e)$ is linearly independent, then

$$
\operatorname{dim} C_{T}(e)=\operatorname{dim} T-|\operatorname{supp}(e)| .
$$

Proof. Suppose that $\operatorname{supp}(e)$ is linearly independent. Then

$$
\operatorname{dim} \operatorname{Ad}(T)(e)=|\operatorname{supp}(e)|
$$

(see [Goodwin 2005a, Lemma 3.2]). The desired equality follows.

The following is a standard consequence of orbit maps.

Lemma 3.17. Let $e^{\prime} \in \mathfrak{g} \geqslant 2$. Then

$$
\operatorname{dim} C_{B_{L}}\left(e^{\prime}\right) \leqslant \operatorname{dim} \mathfrak{c}_{\mathfrak{b}_{L}}\left(e^{\prime}\right) \text { and } \operatorname{dim} C_{U_{L}}\left(e^{\prime}\right) \leqslant \operatorname{dim} \mathfrak{c}_{\mathfrak{n}}\left(e^{\prime}\right) .
$$

Goodwin [2006b, Proposition 5.4] showed that each $U$-orbit in $\mathfrak{u}$ admits a unique so-called minimal orbit representative; see [loc. cit., Definition 5.3]. (This depends on a suitable choice of an ordering of the positive roots compatible with the height function [loc. cit., Definition 3.1]. Moreover, a special case of [loc. cit., Proposition 7.7] gives

$$
C_{B}(e)=C_{T}(e) C_{U}(e)
$$

for $e$ the minimal representative of its $U$-orbit in $\mathfrak{u}$. Hence:

Lemma 3.18. Let $e^{\prime} \in \mathfrak{g}_{\geqslant 2}$. Suppose that $e^{\prime}$ is the minimal representative of its $U$-orbit in $\mathfrak{u}$. Then $C_{B_{L}}\left(e^{\prime}\right)=C_{T}\left(e^{\prime}\right) C_{U_{L}}\left(e^{\prime}\right)$. In particular,

$$
\operatorname{dim} C_{B_{L}}\left(e^{\prime}\right)=\operatorname{dim} C_{T}\left(e^{\prime}\right)+\operatorname{dim} C_{U_{L}}\left(e^{\prime}\right) .
$$

Proposition 3.19. Let $G$ be a simple algebraic group. Table 2 gives a complete list of the height 3 nilpotent orbits in $\mathfrak{g}$. 


\begin{tabular}{cc} 
Type of $G$ & Orbits \\
\hline$A_{n}$ & - \\
$B_{n}$ & {$\left[1^{j}, 2^{2 i}, 3\right]$ with $i>0$} \\
$C_{n}$ & - \\
$D_{n}$ & {$\left[1^{j}, 2^{2 i}, 3\right]$ with $i>0$}
\end{tabular}

\begin{tabular}{cc} 
Type of $G$ & Orbits \\
\hline$G_{2}$ & $\widetilde{A_{1}}$ \\
$F_{4}$ & $A_{1}+\widetilde{A_{1}}$ \\
$E_{6}$ & $3 A_{1}$ \\
$E_{7}$ & $\left(3 A_{1}\right)^{\prime}, 4 A_{1}$ \\
$E_{8}$ & $3 A_{1}, 4 A_{1}$
\end{tabular}

Table 2. The nilpotent orbits of height 3 .

Proof. For the classical groups we use Proposition 2.26. By Remarks 2.27, there are no height 3 nilpotent orbits in types $A_{n}$ and $C_{n}$. Using the tables in [Carter 1985, $\S 13]$ and (2-4), one readily determines the desired orbits when $G$ is exceptional.

In Table 2 we either give the partition or the Bala-Carter label of the corresponding orbit; see [Carter 1985, §13].

In the next three sections we concentrate on the height 3 orbits in types $B_{n}, D_{n}$, and the exceptional types, respectively.

3.5. Height three nilpotent elements of $\mathfrak{s o}_{2 n+1}(\boldsymbol{k})$. Let $G$ be of type $B_{n}$ for $n \geqslant 3$, so $\mathfrak{g}=\mathfrak{s o}_{2 n+1}(k)$. The nilpotent orbits in $\mathfrak{g}$ are classified by the partitions of $2 n+1$ with even parts occurring with even multiplicity [Jantzen 2004, Theorem 1.6]. By Proposition 2.26, the height 3 nilpotent orbits correspond to partitions of $2 n+1$ of the form $\pi_{r, s}=\left[1^{s}, 2^{2 r}, 3\right]$, where $r \geqslant 1, s \geqslant 0$ and $2 r+s+1=n$. Denote the corresponding nilpotent orbit by $\mathrm{O}_{r, s}$ and a representative of such an orbit by $e_{r, s}$. Lemma 3.20. There are precisely $\left[\frac{n-1}{2}\right]$ distinct height 3 nilpotent orbits in $\mathfrak{g}$.

Proof. By our comments above, we need to show that there are precisely $\left[\frac{n-1}{2}\right]$ partitions of $2 n+1$ of the form $\pi_{r, s}$. This is equivalent to finding all partitions of $n-1$ of the form $\left[1^{\frac{s}{2}}, 2^{r}\right]$. Thus $r$ satisfies $1 \leqslant r \leqslant \frac{n-1}{2}$. Since $r$ is an integer, the result follows.

Since the number $2 r+1$ appears frequently in the sequel, we set $\hat{r}=2 r+1$. Using [Carter 1985, §13], we readily see that that $e_{r, s}$ has the weighted Dynkin diagram as in Figure 1.

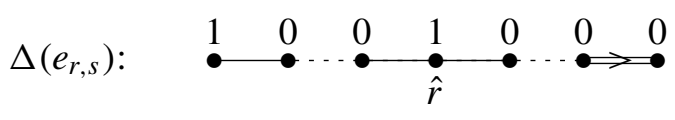

Figure 1. Labeling of $\Delta\left(e_{r, s}\right)$.

Remark 3.21. Note that in $\Delta\left(e_{r, s}\right)$ there are precisely two simple roots, $\alpha_{1}$ and $\alpha_{\hat{r}}$ that are labeled with a " 1 " and that there is an odd number of simple roots between $\alpha_{1}$ and $\alpha_{\hat{r}}$. Also, the short simple root is labeled with a " 1 " if and only if $s=0$, and this can only happen when $n$ is odd. 
We refer to [Bourbaki 1968, Planche II] for information regarding the root system of type $B_{n}$. Let $\alpha_{1}, \ldots, \alpha_{n}$ be the simple roots of $\Psi^{+}$and let

$$
\begin{aligned}
& \beta_{j, k}=\alpha_{j}+\cdots+\alpha_{k} \quad \text { for } 1 \leqslant j \leqslant k \leqslant n, \\
& \gamma_{j, k}=\alpha_{j}+\cdots+\alpha_{k-1}+2 \alpha_{k}+\cdots+2 \alpha_{n} \quad \text { for } 1 \leqslant j<k<n,
\end{aligned}
$$

where $\beta_{j, j}=\alpha_{j}$. Note that all the possible $\beta$ 's and $\gamma$ 's exhaust $\Psi^{+}$.

For a $T$-stable Lie subalgebra $\mathfrak{m}$ of $\mathfrak{u}$, recall the definition of the set of roots $\Psi(\mathfrak{m})$ of $\mathfrak{m}$ with respect to $T$ from Section 2.1.

Lemma 3.22. For an associated cocharacter of $e_{r, s}$ in $\mathfrak{g}$ we have

(i) $\Psi(\mathfrak{g}(2))=\left\{\beta_{1, j}, \gamma_{i, m}, \gamma_{l, k} \mid 1<l<k \leqslant \hat{r} \leqslant j\right.$ and $\left.1<i<m \leqslant \hat{r}\right\}$, and so $\operatorname{dim} \mathfrak{g}(2)=2 r^{2}-r+2 s+1$.

(ii) $\Psi(\mathfrak{g}(3))=\left\{\gamma_{1, k} \mid k \leqslant \hat{r}\right\}$, and so $\operatorname{dim} \mathfrak{g}(3)=2 r$.

Proof. For every $\delta \in \Psi$ we have that $\mathfrak{g}_{\delta} \subseteq \mathfrak{g}(i)$ for some $i \in\{0, \pm 1, \pm 2, \pm 3\}$. For the simple roots this information can be read off from $\Delta\left(e_{r, s}\right)$; see (2-3). Let $\delta=\sum_{\alpha \in \Pi} c_{\delta, \alpha} \alpha$ be a positive root.

Now $\mathfrak{g}_{\delta} \subseteq \mathfrak{g}(2)$ if and only if $c_{\delta, \alpha_{1}}+c_{\delta, \alpha_{\hat{r}}}=2$. All of the roots listed above satisfy this condition, and no others do. Finally, $\mathfrak{g}_{\delta} \subseteq \mathfrak{g}(3)$ if and only if $c_{\delta, \alpha_{1}}+c_{\delta, \alpha_{\hat{r}}}=3$. All of the roots listed above satisfy this condition, and no others do.

Lemma 3.23. For an associated cocharacter of $e_{r, s}$ in $\mathfrak{g}$ we have

(i) $\Psi\left(\mathfrak{b}_{L}\right)=\left\{\beta_{j, k}, \gamma_{l, m} \mid \hat{r}<j\right.$ or $\left.1<j \leqslant k<\hat{r}, \hat{r}<l<m\right\}$.

(ii) $\operatorname{dim} \mathfrak{b}_{L}=2 r^{2}+s^{2}+s+r+1$.

Proof. For every $\delta \in \Psi$ we have that $\mathfrak{g}_{\delta} \subseteq \mathfrak{g}(i)$ for some $i \in\{0, \pm 1, \pm 2, \pm 3\}$. As mentioned above, for the simple roots this information can be read off from $\Delta\left(e_{r, s}\right)$; see (2-3). Let $\delta=\sum_{\alpha \in \Pi} c_{\delta, \alpha} \alpha \in \Psi^{+}$. Then $\mathfrak{g}_{\delta} \subseteq \mathfrak{b}_{L}$ if and only if $c_{\delta, \alpha_{1}}+c_{\delta, \alpha_{\hat{r}}}=0$. All of the roots listed above satisfy this condition, and no others do. Consequently, $\operatorname{dim} \mathfrak{n}=2 r^{2}+s^{2}-r$. Since $\operatorname{dim} \mathfrak{t}=n$, we get $\operatorname{dim} \mathfrak{b}_{L}=2 r^{2}+s^{2}+s+r+1$.

It follows from Figure 1 that $L$ is of Dynkin type $A_{\hat{r}-1} \times B_{S}$. Accordingly, there is a natural partition of the roots of $\mathfrak{b}_{L}$ into a union of two subsets, namely the positive roots of the $A_{\hat{r}-1}$ and $B_{S}$ subsystems, respectively. Thus, we have $\Psi\left(\mathfrak{b}_{L}\right)=\Psi_{1}\left(\mathfrak{b}_{L}\right) \cup \Psi_{2}\left(\mathfrak{b}_{L}\right)$, where

$$
\begin{aligned}
\Psi_{1}\left(\mathfrak{b}_{L}\right) & =\left\{\beta_{j, k} \mid 1<j \leqslant k<\hat{r}\right\}, \\
\Psi_{2}\left(\mathfrak{b}_{L}\right) & =\left\{\beta_{j, k}, \gamma_{l, m} \mid \hat{r}<j \leqslant k, \hat{r}<l<m\right\} .
\end{aligned}
$$

Similarly, we can decompose the roots of $\mathfrak{g}_{\geqslant 2}$ into two sets as follows: $\Psi\left(\mathfrak{g}_{\geqslant 2}\right)=$ $\Psi_{1}\left(\mathfrak{g}_{\geqslant 2}\right) \cup \Psi_{2}\left(\mathfrak{g}_{\geqslant 2}\right)$, where

$$
\begin{aligned}
& \Psi_{1}\left(\mathfrak{g}_{\geqslant 2}\right)=\left\{\gamma_{j, k} \mid 1 \leqslant j<k \leqslant \hat{r}\right\}, \\
& \Psi_{2}\left(\mathfrak{g}_{\geqslant 2}\right)=\left\{\beta_{1, j}, \gamma_{1, k} \mid \hat{r} \leqslant j, \hat{r}<k\right\} .
\end{aligned}
$$


The sets $\Psi_{i}\left(\mathfrak{b}_{L}\right)$ and $\Psi_{i}\left(\mathfrak{g}_{\geqslant 2}\right)$ satisfy the following property:

$$
\delta \in \Psi_{i}\left(\mathfrak{b}_{L}\right), \eta \in \Psi_{3-i}\left(\mathfrak{g}_{\geqslant 2}\right) \Rightarrow \delta+\eta \notin \Psi, i \in\{1,2\} .
$$

Denote by $\mathfrak{b}_{L}^{i}$ the Lie subalgebras of $\mathfrak{b}_{L}$ such that $\Psi\left(\mathfrak{b}_{L}^{i}\right)=\Psi_{i}\left(\mathfrak{b}_{L}\right)$ for $i=1,2$. For the rest of this section we show that the following element is a representative of the dense $B_{L}$-orbit in $\mathfrak{g}_{\geqslant 2}$; set

$$
e_{r, s}^{\prime}:=\sum_{j, k=0}^{r-1}\left(e_{\gamma_{\hat{r}-2 j-1, \hat{r}-2 j}}+e_{\gamma_{1, \hat{r}-2 k}}\right)+e_{\gamma_{1, \hat{r}+1}}+e_{\beta_{1, \hat{r}}},
$$

where $e_{\delta} \in \mathfrak{g}_{\delta} \backslash\{0\}$ for $\delta \in \Psi\left(\mathfrak{g}_{\geqslant 2}\right)$.

Recall from the paragraph before Lemma 3.18 the notion of minimal $U$-orbit representatives in $\mathfrak{u}$ from [Goodwin 2006b].

Lemma 3.24. Each $e_{r, s}^{\prime}$ is the minimal representative of its $U$-orbit in $\mathfrak{u}, \operatorname{supp}\left(e_{r, s}^{\prime}\right)$ is linearly independent, and

$$
\left|\operatorname{supp}\left(e_{r, s}^{\prime}\right)\right|= \begin{cases}2 r+2 & \text { if } s>0 \\ 2 r+1 & \text { if } s=0 .\end{cases}
$$

Proof. It is straightforward to check that $e_{r, s}^{\prime}$ is the minimal representative of its $U$ orbit in $\mathfrak{u}$ in the sense of [Goodwin 2006b] and one easily computes $\left|\operatorname{supp}\left(e_{r, s}^{\prime}\right)\right|$. Note that the root $\gamma_{1, \hat{r}+1}$ only occurs if $s>0$.

Suppose there exist scalars $\tau_{j}, \xi_{k}, \mu$ and $v$ such that

$$
\sum_{j=0}^{r-1} \tau_{j} \gamma_{\hat{r}-2 j-1, \hat{r}-2 j}+\sum_{k=0}^{r-1} \xi_{k} \gamma_{1, \hat{r}-2 k}+\mu \gamma_{1, \hat{r}+1}+\nu \beta_{1, \hat{r}}=0 .
$$

Since the coefficients of $\alpha_{1}, \alpha_{2}$, and $\alpha_{3}$ must be zero, we have

$\sum_{k=0}^{r-1} \xi_{k}+\mu+v=0, \tau_{r-1}+\sum_{k=0}^{r-1} \xi_{k}+\mu+v=0$, and $\xi_{r-1}+2 \tau_{r-1}+\sum_{k=0}^{r-1} \xi_{k}+\mu+v=0$.

These three equations imply that $\tau_{r-1}=0=\xi_{r-1}$. Continuing in this way, we see that $\tau_{j}=0=\xi_{j}$ for all $j$. Thus we are left to show that $\gamma_{1, \hat{r}+1}$ and $\beta_{1, \hat{r}}$ are linearly independent but this is obvious.

Thanks to Lemma 3.24 it is harmless to assume that $\operatorname{supp}\left(e_{r, s}^{\prime}\right)$ is part of a Chevalley basis of $\mathfrak{g}$.

Lemma 3.25. $\operatorname{dim} \mathfrak{c}_{\mathfrak{n}}\left(e_{r, s}^{\prime}\right)=\left\{\begin{array}{cl}(s-1)^{2} & \text { if } s>0 \\ 0 & \text { if } s=0\end{array}\right.$ 
Proof. Thanks to (3-3), we may consider the two summands

$$
\sum_{j, k=0}^{r-1}\left(e_{\gamma_{\hat{r}-2 j-1, \hat{r}-2 j}}+e_{\gamma_{1, \hat{r}-2 k}}\right) \quad \text { and } \quad e_{\gamma_{1, \hat{r}+1}}+e_{\beta_{1, \hat{r}}}
$$

of $e_{r, S}^{\prime}$ separately. Since $\gamma_{\hat{r}-2 j-1, \hat{r}-2 j}+\gamma_{1, \hat{r}-2 k} \in \Psi_{1}\left(\mathfrak{g}_{\geqslant 2}\right)$, we need only consider the root spaces $\mathfrak{g}_{\delta}$ for $\delta \in \Psi_{1}\left(\mathfrak{b}_{L}\right)$. So let $\beta_{i, m} \in \Psi_{1}\left(\mathfrak{b}_{L}\right)$. If $m=\hat{r}-2 l$ for some $0 \leqslant l<r$, then, by the Chevalley commutator relations

$$
\left[e_{\gamma_{\hat{r}-2 l+1, \hat{r}-2(l-1)}}, \mathfrak{g}_{\beta_{i, \hat{r}-2 l}}\right]=\mathfrak{g}_{\gamma_{i, \hat{r}-2(l-1)}},
$$

since char $k$ is good for $G$. If $m=\hat{r}-2 l-1$ for some $0 \leqslant l<r$, then

$$
\left[e_{\gamma_{1, \hat{r}-2 l}}, \mathfrak{g}_{\beta_{i, \hat{r}-2 l-1}}\right]=\mathfrak{g}_{\gamma_{1, i}} .
$$

Next we observe that all the $\beta$ 's above exhaust the set $\Psi_{1}\left(\mathfrak{b}_{L}\right)$. Consequently,

$$
\mathfrak{c}_{\mathfrak{b}_{L}^{1}}\left(\sum_{j, k=0}^{r-1}\left(e_{\gamma_{\hat{r}-2 j-1, \hat{r}-2 j}}+e_{\gamma_{1, \hat{r}-2 k}}\right)\right)=\{0\} .
$$

Next we consider the summand $e_{\gamma_{1, \hat{r}+1}}+e_{\beta_{1, \hat{r}}}$. First observe that $\left[\mathfrak{n}, e_{\gamma_{1, \hat{r}+1}}\right]=\{0\}$, so $\mathfrak{c}_{\mathfrak{n}}\left(e_{\gamma_{1, \hat{r}+1}}\right)=\mathfrak{n}$. Second the root $\beta_{1, \hat{r}}$ lies in $\Psi_{2}(\mathfrak{g} \geqslant 2)$. Thanks to property (3-3), we need only consider roots $\delta \in \Psi_{2}\left(\mathfrak{b}_{L}\right)$. We see that the only roots $\delta \in \Psi_{2}\left(\mathfrak{b}_{L}\right)$ with $\delta+\beta_{1, \hat{r}} \in \Psi\left(\mathfrak{g}_{\geqslant 2}\right)$ are of the form $\beta_{\hat{r}+1, j}$ or $\gamma_{\hat{r}+1, k}$ where $\hat{r}+1 \leqslant j \leqslant n$ and $\hat{r}+1<k \leqslant n$. Again the Chevalley commutator relations imply

$$
\left[\mathfrak{g}_{\beta_{\hat{r}+1, j}}, e_{\beta_{1, \hat{r}}}\right]=\mathfrak{g}_{\beta_{1, j}}, \quad \text { and } \quad\left[\mathfrak{g}_{\gamma_{\hat{r}+1, k}}, e_{\beta_{1, \hat{r}}}\right]=\mathfrak{g}_{\gamma_{1, k}} .
$$

We also observe that $\beta_{j, k}$ and $\gamma_{l, m}$ for $\hat{r}+1<j, l$ have the property that

$$
\beta_{1, \hat{r}+1}+\gamma_{l, m}, \beta_{1, \hat{r}+1}+\beta_{j, k} \notin \Psi_{2}\left(\mathfrak{g}_{\geqslant 2}\right) .
$$

All the roots above exhaust $\Psi_{2}\left(\mathfrak{b}_{L}\right)$, so we conclude that all the roots $\beta_{j, k}$ and $\gamma_{l, m}$ for $\hat{r}+1<j, l$ of $\Psi_{2}\left(\mathfrak{b}_{L}\right)$ are all contained in $\Psi\left(\mathfrak{c}_{\mathfrak{n}}\left(e_{\beta_{1, \hat{r}}}\right)\right)$. If $s>0$, these roots form the set of positive roots of a root system of type $B_{s-1}$, there are exactly $(s-1)^{2}$ positive roots in a root system of type $B_{s-1}$ and so $\left|\Psi\left(\mathfrak{c}_{\mathfrak{n}}\left(e_{\beta_{1, \hat{r}}}\right)\right)\right|=(s-1)^{2}$. Therefore, $\operatorname{dim} \mathfrak{c}_{\mathfrak{n}}\left(e_{r, s}^{\prime}\right)=(s-1)^{2}$; clearly, if $s=0$ then $\operatorname{dim} \mathfrak{c}_{\mathfrak{n}}\left(e_{r, s}^{\prime}\right)=0$.

Proposition 3.26. The $B_{L}$-orbit of $e_{r, s}^{\prime}$ is dense in $\mathfrak{g}_{\geqslant 2}$.

Proof. Thanks to Lemma 3.15, it is sufficient to show that

$$
\operatorname{dim} B_{L}=\operatorname{dim} C_{B_{L}}\left(e_{r, s}^{\prime}\right)+\operatorname{dim} \mathfrak{g}_{\geqslant 2} .
$$

Lemma 3.22 implies that $\operatorname{dim} \mathfrak{g} \geqslant 2=2 r^{2}+2 s+r+1$ and Lemma 3.23 implies that $\operatorname{dim} B_{L}=2 r^{2}+s^{2}+s+r+1$. By Lemma 3.24, $e_{r, s}^{\prime}$ is the minimal representative 
of its $U$-orbit in $\mathfrak{u}$. Thus, by Lemma 3.18, we have

$$
\operatorname{dim} C_{B_{L}}\left(e_{r, s}^{\prime}\right)=\operatorname{dim} C_{T}\left(e_{r, s}^{\prime}\right)+\operatorname{dim} C_{U}\left(e_{r, s}^{\prime}\right) .
$$

Consequently, Lemmas 3.17, 3.24, and 3.25 imply that, for $s>0, \operatorname{dim} C_{B_{L}}\left(e_{r, s}^{\prime}\right) \leqslant$ $n-2 r-2+(s-1)^{2}=s^{2}-s$. So

$$
\operatorname{dim} C_{B_{L}}\left(e_{r, s}^{\prime}\right)+\operatorname{dim} \mathfrak{g}_{\geqslant 2} \leqslant s^{2}-s+2 r^{2}+r+2 s+1=\operatorname{dim} B_{L} .
$$

This clearly implies $\operatorname{dim} B_{L}=\operatorname{dim} C_{B_{L}}\left(e_{r, s}^{\prime}\right)+\operatorname{dim} \mathfrak{g}_{\geqslant 2}$. Similarly, if $s=0$, we get $\operatorname{dim} B_{L}=\operatorname{dim} C_{B_{L}}\left(e_{r, s}^{\prime}\right)+\operatorname{dim} \mathfrak{g}_{\geqslant 2}$.

Corollary 3.27. $\operatorname{dim} C_{B_{L}}\left(e_{r, s}^{\prime}\right)=s(s-1)$.

Finally, from Lemma 3.15 we obtain

Corollary 3.28. If $G$ is of type $B_{n}$ and $e \in \mathcal{N}$ with $\mathrm{ht}(e)=3$, then e is spherical.

3.6. Height three nilpotent elements of $\mathfrak{s o}_{2 n}(\boldsymbol{k})$. Assume now that $G$ is of type $D_{n}$ for $n \geqslant 4$, so $\mathfrak{g}=\mathfrak{s o}_{2 n}$. We know that the nilpotent orbits in $\mathfrak{g}$ are classified by the partitions of $2 n$ with even parts occurring with even parity; see [Jantzen 2004, Theorem 1.6]. We showed that the height three nilpotent orbits correspond to partitions of $2 n$ of the form $\pi_{r, s}=\left[1^{2 s+1}, 2^{2 r}, 3\right]$ where $r \geqslant 1, s \geqslant 0$ and $2 r+s+2=$ $n$; see Proposition 2.26. Similarly to the $B_{n}$ case, we denote the corresponding orbit by $\mathrm{O}_{r, s}$ and a representative of such an orbit by $e_{r, s}$. Because the proofs of the results in this section are virtually identical to the ones in Section 3.5, they are omitted.

Lemma 3.29. There are precisely $\left[\frac{n-2}{2}\right]$ distinct height 3 nilpotent orbits in $\mathfrak{g}$.

Using [Carter 1985, §13], we can easily calculate that for $s>0, e_{r, s}$ has the weighted Dynkin diagram $\Delta\left(e_{r, s}\right)$ as shown in Figure 2.

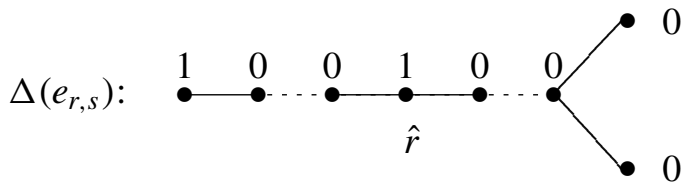

Figure 2. Labelling of $\Delta\left(e_{r, s}\right)$ for $s>0$.

Similarly, when $s=0$, the labelling of $\Delta\left(e_{r, 0}\right)$ is shown in Figure 3.

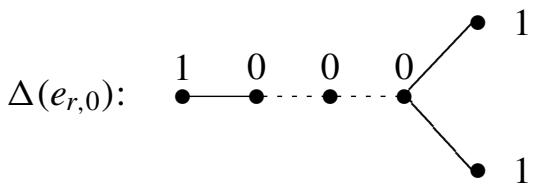

Figure 3. Labelling of $\Delta\left(e_{r, 0}\right)$. 
Remark 3.30. Note that there is always an odd number of " 0 " labels between the first and second " 1 " labels in $\Delta\left(e_{r, s}\right)$. If $s>0$, then there are $s+1$ " 0 " labels to the right of the second " 1 " label. Finally, $s=0$ only if $n$ is even.

We refer to [Bourbaki 1968, Planche IV] for information regarding the root system of type $D_{n}$. We use the following notation for the positive roots $\Psi^{+}$. Let $\alpha_{1}, \ldots, \alpha_{n}$ be the set of simple roots of $\Psi^{+}$and let

$$
\begin{aligned}
\beta_{j, k} & =\alpha_{j}+\cdots+\alpha_{k} \quad \text { for } 1 \leqslant j \leqslant k \leqslant n \text { not } j=n-1, k=n, \\
\beta_{j} & =\alpha_{j}+\cdots+\alpha_{n-2}+\alpha_{n} \text { for } 1 \leqslant j \leqslant n-2, \\
\gamma_{j, k} & =\alpha_{j}+\cdots+\alpha_{k-1}+2 \alpha_{k}+\cdots+2 \alpha_{n-2}+\alpha_{n-1}+\alpha_{n} \text { for } 1 \leqslant j<k<n-2 .
\end{aligned}
$$

Here we again use the convention $\beta_{j, j}=\alpha_{j}$. Note that all the possible $\beta$ 's and $\gamma$ 's exhaust $\Psi^{+}$.

Next we consider the structure of the abelian Lie subalgebra $\mathfrak{g} \geqslant 2=\mathfrak{g}(2) \oplus \mathfrak{g}(3)$.

Lemma 3.31. An associated cocharacter for $e_{r, s}$ affords the following.

(i) $\Psi(\mathfrak{g}(2))=\left\{\begin{array}{ll}\left\{\beta_{1, j}, \beta_{1}, \gamma_{l, k}, \gamma_{1, m} \mid 1<l<k \leqslant \hat{r} \leqslant j, \hat{r}<m\right\} & \text { if } s>0, \\ \left\{\beta_{1, n-1}, \beta_{1}, \beta_{i, n}, \gamma_{j, k} \mid 2 \leqslant i<\hat{r}, 1<j<k<\hat{r}\right\} & \text { if } s=0 .\end{array}\right.$ In particular, $\operatorname{dim} \mathfrak{g}(2)=2 r^{2}-r+2 s+2$.

(ii) $\Psi(\mathfrak{g}(3))=\left\{\begin{array}{ll}\left\{\gamma_{1, k} \mid k \leqslant \hat{r}\right\} & \text { if } s>0, \\ \left\{\beta_{1, n}, \gamma_{1, k} \mid 2 \leqslant k<\hat{r}\right\} & \text { if } s=0 .\end{array}\right.$ In particular, $\operatorname{dim} \mathfrak{g}(3)=2 r$.

Next we look at the structure of the Lie subalgebra $\mathfrak{b}_{L}$ of $\mathfrak{g}(0)$.

Lemma 3.32. An associated cocharacter for $e_{r, s}$ affords the following.

$$
\Psi\left(\mathfrak{b}_{L}\right)= \begin{cases}\left\{\beta_{i}, \beta_{j, k}, \gamma_{l, m} \mid \hat{r}<j \text { or } 1<j \leqslant k<\hat{r}, \hat{r}<i, \hat{r}<l<m\right\} & \text { if } s>0, \\ \left\{\beta_{j, k} \mid 1<j \leqslant k<\hat{r}\right\} & \text { if } s=0 .\end{cases}
$$

In particular, $\operatorname{dim} \mathfrak{b}_{L}=2 r^{2}+s^{2}+r+2 s+2$.

Similarly to the $B_{n}$ case, the roots of $\mathfrak{b}_{L}$ naturally form two distinct subsets, namely the roots whose support lies strictly to the left of the second " 1 " label of the weighted Dynkin diagram and those whose support lies strictly to the right of the second " 1 " label of the weighted Dynkin diagram. More precisely, we have $\Psi\left(\mathfrak{b}_{L}\right)=\Psi_{1}\left(\mathfrak{b}_{L}\right) \cup \Psi_{2}\left(\mathfrak{b}_{L}\right)$ where

$$
\begin{aligned}
\Psi_{1}\left(\mathfrak{b}_{L}\right) & =\left\{\beta_{j, k} \mid 1<j \leqslant k<\hat{r}\right\}, \\
\Psi_{2}\left(\mathfrak{b}_{L}\right) & =\left\{\beta_{j, k}, \beta_{i}, \gamma_{l, m} \mid \hat{r}<j \leqslant k, \hat{r}<i, \hat{r}<l<m\right\} .
\end{aligned}
$$

Again we partition the roots of $\mathfrak{g}_{\geqslant 2}$ into two distinct subsets. More precisely, we write $\Psi\left(\mathfrak{g}_{\geqslant 2}\right)=\Psi_{1}\left(\mathfrak{g}_{\geqslant 2}\right) \cup \Psi_{2}\left(\mathfrak{g}_{\geqslant 2}\right)$, where for $s \geqslant 1$, we define

$$
\begin{aligned}
& \Psi_{1}\left(\mathfrak{g}_{\geqslant 2}\right)=\left\{\gamma_{j, k} \mid 1 \leqslant j<k \leqslant \hat{r}\right\}, \\
& \Psi_{2}\left(\mathfrak{g}_{22}\right)=\left\{\beta_{1}, \beta_{1, j}, \gamma_{1, k} \mid \hat{r} \leqslant j, \hat{r}<k\right\},
\end{aligned}
$$


and for $s=0$, we define

$$
\begin{aligned}
& \Psi_{1}\left(\mathfrak{g}_{\geqslant 2}\right)=\left\{\gamma_{j, k} \mid 1 \leqslant j<k \leqslant \hat{r}\right\}, \\
& \Psi_{2}\left(\mathfrak{g}_{\geqslant 2}\right)=\left\{\beta_{1}, \beta_{1, n-1}, \beta_{j, n} \gamma_{1, k} \mid j \leqslant \hat{r}<k\right\} .
\end{aligned}
$$

Again, we have the following property of these sets:

$$
\delta \in \Psi_{i}\left(\mathfrak{b}_{L}\right), \eta \in \Psi_{3-i}\left(\mathfrak{g}_{\geqslant 2}\right) \Rightarrow \delta+\eta \notin \Psi, i \in\{1,2\} .
$$

For $s>1$, set

$$
e_{r, s}^{\prime}:=\sum_{j, k=0}^{r-1}\left(e_{\gamma_{\hat{r}-2 j-1, \hat{r}-2 j}}+e_{\gamma_{1, \hat{r}-2 k}}\right)+e_{\gamma_{1, \hat{r}+1}}+e_{\beta_{1, \hat{r}}} \in \mathfrak{g}_{\geqslant 2},
$$

for $s=1$, set

$$
e_{r, 1}^{\prime}:=\sum_{j, k=0}^{r-1}\left(e_{\gamma_{\hat{r}-2 j-1, \hat{r}-2 j}}+e_{\gamma_{1, \hat{r}-2 k}}\right)+e_{\beta_{1, n}}+e_{\beta_{1, \hat{r}}} \in \mathfrak{g}_{\geqslant 2},
$$

and for $s=0$, set

$$
e_{r, 0}^{\prime}:=\sum_{j, k=1}^{r-1}\left(e_{\gamma_{\hat{r}-2 j-1, \hat{r}-2 j}}+e_{\gamma_{1, \hat{r}-2 k}}\right)+e_{\beta_{1, n}}+e_{\beta_{1, n-1}}+e_{\beta_{n-2, n}}+e_{\beta_{1}} \in \mathfrak{g}_{\geqslant 2} .
$$

Lemma 3.33. With the notation as above, we have $\left|\operatorname{supp}\left(e_{r, s}^{\prime}\right)\right|=2 r+2, \operatorname{supp}\left(e_{r, s}^{\prime}\right)$ is linearly independent, and $\operatorname{dim} \mathfrak{c}_{\mathfrak{n}}\left(e_{r, s}^{\prime}\right)=s(s-1)$.

Proposition 3.34. The $B_{L}$-orbit of $e_{r, s}^{\prime}$ is dense in $\mathfrak{g}_{2}$.

Corollary 3.35. If $G$ is of type $D_{n}$ and $e \in \mathcal{N}$ with ht $(e)=3$, then e is spherical.

3.7. Height three nilpotent elements of the exceptional Lie algebras. We fix an ordering of the roots $\alpha_{1}, \ldots, \alpha_{r}$ of $\Psi(\mathfrak{g} \geqslant 2)$ such that $\alpha_{i} \prec \alpha_{j}$ for $i<j$. Define the subalgebra $\mathfrak{m}_{i}$ of $\mathfrak{g}_{2}$ by setting

$$
\mathfrak{m}_{i}=\bigoplus_{j=i+1}^{r} \mathfrak{g}_{\alpha_{j}}
$$

and the quotient $\mathfrak{q}_{i}$ by $\mathfrak{q}_{i}=\mathfrak{g}_{\geqslant 2} / \mathfrak{m}_{i}$ for $0 \leqslant i \leqslant r$. Let $B$ be a Borel subgroup of $G$ such that $\mathfrak{g}_{\geqslant 2} \subseteq$ Lie $R_{u}(B)=\mathfrak{u}$. Note that each $\mathfrak{q}_{i}$ is a $B$-module.

The computer programme, DOOBS, devised by S. M. Goodwin allows us to determine whether $B$ acts on $\mathfrak{g} \geqslant 2$ with a dense orbit. For details of the GAP4 [Gap 2004] computer algebra program, we refer the reader to [Goodwin 2005a; 2006a]. Working inductively, starting with $i=0$, at each stage of the algorithm, DOOBS determines a representative $x_{i}+\mathfrak{m}_{i}$, with $\operatorname{supp}\left(x_{i}\right)$ linearly independent of a dense $B$-orbit on $\mathfrak{q}_{i}$ or decides that $B$ does not act on $\mathfrak{q}_{i}$ with a dense orbit. 
DOOBS also keeps a record of the primes for which

$$
\operatorname{dim}_{p} \mathfrak{c}_{\mathfrak{u}}\left(x_{i}+\mathfrak{m}_{i+1}\right)>\operatorname{dim}_{0} \mathfrak{c}_{\mathfrak{u}}\left(x_{i}+\mathfrak{m}_{i+1}\right),
$$

where $\operatorname{dim}_{p} \mathfrak{c}_{\mathfrak{u}}\left(x_{i}+\mathfrak{m}_{i+1}\right)$ and $\operatorname{dim}_{0} \mathfrak{c}_{\mathfrak{u}}\left(x_{i}+\mathfrak{m}_{i+1}\right)$ denote the dimension of $\mathfrak{c}_{\mathfrak{u}}\left(x_{i}+\right.$ $\mathfrak{m}_{i+1}$ ) over a field of characteristic $p$ and characteristic 0 respectively [Goodwin 2005a, Remark 3.2]. For these primes we cannot conclude that $B$ acts on $\mathfrak{g} \geqslant 2$ with a dense orbit. If DOOBS determines that $B$ acts on $\mathfrak{g} \geqslant 2$ with a dense orbit, then it calculates a representative of the dense orbit and a list of primes for which the result is not necessarily valid.

There is a variant of DOOBS called DOOBSLevi [Goodwin 2006a]. This program considers a parabolic subgroup $P=L R_{u}(P)$ and determines whether a Borel subgroup $B_{L}$ of $L$ acts on an ideal of Lie $R_{u}(P)$ with a dense orbit. The algorithm used to determine whether $B_{L}$ acts on an ideal with a dense orbit is essentially the same as the DOOBS algorithm, with $B_{L}$ replacing $B$. DOOBSLevi also records the primes for which its conclusions are not necessarily valid.

Let $e \in \mathcal{N}$ of height 3 and let $\lambda$ be a cocharacter of $G$ that is associated to $e$. We use the same numbering of the positive roots as in GAP4. Table 3 lists the roots whose root subgroups together with $T$ generate the Levi subgroup $C_{G}(\lambda)$ and we also list the roots whose root subspaces generate $\mathfrak{g} \geqslant 2$ (as a $B$-submodule of $\mathfrak{g}$ ) for the 7 cases of height three nilpotent orbits for the simple exceptional groups; see Proposition 3.19. These are determined by means of the weighted Dynkin diagrams.

\begin{tabular}{cccc} 
Type of $G$ & Bala-Carter label & Generators for $L$ & Generators for $\mathfrak{g}_{\geqslant 2}$ \\
\hline$G_{2}$ & $\widetilde{A_{1}}$ & $\alpha_{2}$ & $\alpha_{4}$ \\
$F_{4}$ & $A_{1}+\widetilde{A_{1}}$ & $\Pi \backslash\left\{\alpha_{4}\right\}$ & $\alpha_{16}$ \\
$E_{6}$ & $3 A_{1}$ & $\Pi \backslash\left\{\alpha_{4}\right\}$ & $\alpha_{24}$ \\
$E_{7}$ & $\left(3 A_{1}\right)^{\prime}$ & $\Pi \backslash\left\{\alpha_{3}\right\}$ & $\alpha_{37}$ \\
$E_{7}$ & $4 A_{1}$ & $\Pi \backslash\left\{\alpha_{2}, \alpha_{7}\right\}$ & $\alpha_{30}, \alpha_{53}$ \\
$E_{8}$ & $3 A_{1}$ & $\Pi \backslash\left\{\alpha_{7}\right\}$ & $\alpha_{74}$ \\
$E_{8}$ & $4 A_{1}$ & $\Pi \backslash\left\{\alpha_{2}\right\}$ & $\alpha_{69}$
\end{tabular}

Table 3. Height three nilpotent orbits in the exceptional Lie algebras.

The height 3 cases for the exceptional groups were analyzed using DOOBSLevi algorithm. It turns out that there are no characteristic restrictions in these cases.

Lemma 3.36. If $G$ is simple of exceptional type and $e \in \mathcal{N}$ with $\mathrm{ht}(e)=3$, then $e$ is spherical.

Corollaries 3.28 and 3.35 combined with Lemma 3.36 give the following result. 
Proposition 3.37. Let $G$ be a connected reductive algebraic group and let $e \in \mathcal{N}$. If $\operatorname{ht}(e)=3$, then e is spherical.

Proof. If $G$ is simple, then the statement follows from Corollaries 3.28 and 3.35 and Lemma 3.36. In the general case we argue as in Lemma 3.3 to reduce to the simple case.

3.8. The classification. Our main classification theorem now follows readily from Lemma 3.3 and Propositions 3.14 and 3.37.

Theorem 3.38. Let $G$ be a connected reductive algebraic group. Suppose that char $k$ is a good prime for $G$. Then a nilpotent element $e \in \mathfrak{g}$ is spherical if and only if ht $(e) \leqslant 3$.

Remark 3.39. Let $G$ be a simple algebraic group and let char $k$ be a good prime for $G$. Then the spherical nilpotent orbits are given in Table 4. We present the orbits by listing the corresponding partition in the classical cases or by giving the corresponding Bala-Carter label for the exceptional groups.

\begin{tabular}{c|l} 
Type of $G$ & Spherical Orbits \\
\hline$A_{n}$ & {$\left[1^{j}, 2^{i}\right]$} \\
$B_{n}$ & {$\left[1^{j}, 2^{2 i}\right]$, or $\left[1^{j}, 2^{2 i}, 3\right]$ with $i \geqslant 0$} \\
$C_{n}$ & {$\left[1^{2 j}, 2^{i}\right]$} \\
$D_{n}$ & {$\left[1^{j}, 2^{2 i}\right]$, or $\left[1^{j}, 2^{2 i}, 3\right]$ with $i \geqslant 0$} \\
\hline$G_{2}$ & $A_{1}$ or $\widetilde{A_{1}}$ \\
$F_{4}$ & $A_{1}, \widetilde{A_{1}}$, or $A_{1}+\widetilde{A_{1}}$ \\
$E_{6}$ & $A_{1}, 2 A_{1}$, or $3 A_{1}$ \\
$E_{7}$ & $A_{1}, 2 A_{1},\left(3 A_{1}\right)^{\prime},\left(3 A_{1}\right)^{\prime \prime}$, or $4 A_{1}$ \\
$E_{8}$ & $A_{1}, 2 A_{1}, 3 A_{1}$, or $4 A_{1}$
\end{tabular}

Table 4. The spherical nilpotent orbits for $G$ simple.

Remark 3.40. Using the fact that in good characteristic a Springer map affords a bijection between the set of unipotent $G$-conjugacy classes and the set of nilpotent $G$-orbits (see [Springer and Steinberg 1970, III, 3.12] and [Bardsley and Richardson 1985, Corollary 9.3.4]), Theorem 3.38 also gives a classification of the spherical unipotent classes in $G$. Here we define the height of a unipotent element $u$ of $G$ as the height of the image of $u$ in $\mathcal{N}$ under a Springer isomorphism.

\section{Applications and complements}

Here we discuss applications of the main result and some further consequences. 
4.1. Spherical distinguished nilpotent elements. Recall that a nilpotent element $e \in \mathcal{N}$ is distinguished in $\mathfrak{g}$ if every torus contained in $C_{G}(e)$ is contained in the centre of $G$. For now we assume that $G$ is simple, so $e$ is distinguished in $\mathfrak{g}$ if and only if any torus contained in $C_{G}(e)$ is trivial and hence $C_{G}(e)^{\circ}$ is unipotent. Further recall that $\kappa_{G}(G \cdot e)=\kappa_{G}\left(G / C_{G}(e)^{\circ}\right)$ (see (2-2)). Since $C_{G}(e)^{\circ}$ is connected and unipotent, it is contained in the unipotent radical $U$ of a Borel subgroup $B=T U$ of $G$. Let $B^{-}=T U^{-}$be the unique opposite Borel subgroup to $B=T U$ relative to $T$ [Humphreys $1975, \S 26.2]$. Consequently,

$$
B^{-} \cap C_{G}(e)^{\circ} \subseteq B^{-} \cap U=\{1\} .
$$

Thus, by (2-1), we have

$$
\kappa_{G}\left(G / C_{G}(e)^{\circ}\right)=\operatorname{dim} G-\operatorname{dim} C_{G}(e)^{\circ}-\operatorname{dim} B^{-}=\operatorname{dim} U-\operatorname{dim} C_{G}(e),
$$

or equivalently, $\kappa_{G}(G \cdot e)=\left|\Psi^{+}\right|-\operatorname{dim} C_{G}(e)$. We summarize what we have just shown.

Proposition 4.1. Let $e \in \mathcal{N}$ be a distinguished nilpotent element. Then

$$
\kappa_{G}(G \cdot e)=\left|\Psi^{+}\right|-\operatorname{dim} C_{G}(e) .
$$

Remark 4.2. Proposition 4.1 was first observed for a field of characteristic zero [Panyushev 1994, Corollary 2.4].

If $G$ is a simple classical group, then the distinguished nilpotent elements are given as follows [Jantzen 2004, Lemmas 4.1 and 4.2].

Lemma 4.3. Let $e \in \mathcal{N}$ and let $\pi_{e}$ be the corresponding partition of $\operatorname{dim} V$.

(i) If $G=\operatorname{SL}(V)$, then $e$ is distinguished if and only if $\pi_{e}=[\operatorname{dim} V]$.

(ii) If $G=\operatorname{Sp}(V)$, then $e$ is distinguished if and only if $\pi_{e}$ consists only of distinct even parts.

(iii) If $G=\mathrm{SO}(V)$, then $e$ is distinguished if and only if $\pi_{e}$ consists only of distinct odd parts.

Corollary 4.4. If $G=\mathrm{SO}(V)$ and $e \in \mathcal{N}$ is spherical and distinguished, then $\operatorname{ht}(e)=2$.

Proof. Thanks to Proposition 3.19, the height 3 nilpotent elements have partitions of the form $\pi=\left[1^{s}, 2^{2 r}, 3\right]$, where $r>0$. Thus such a partition has even parts and so is not distinguished. So if $e$ is spherical and distinguished, then $\operatorname{ht}(e)=2$.

Proposition 2.26 and Lemma 4.3 imply the following result.

Proposition 4.5. Let $e \in \mathcal{N}$ be distinguished and $\pi_{e}$ be the corresponding partition of $\operatorname{dim} V$.

(i) If $G=\operatorname{SL}(V)$, then $\mathrm{ht}(e)=2$ if and only if $\pi_{e}=[2]$. 
(ii) If $G=\operatorname{Sp}(V)$, then $\mathrm{ht}(e)=2$ if and only if $\pi_{e}=[2]$.

(iii) If $G=\mathrm{SO}(V)$, then $\mathrm{ht}(e)=2$ if and only if $\pi_{e}=[3]$ or $\pi_{e}=[1,3]$.

Theorem 4.6. If $G$ is a simple algebraic group and $e \in \mathcal{N}$ is spherical and distinguished, then $G$ is of type $A_{1}$.

Proof. For $G$ simple classical, Proposition 4.5 implies that $G$ is of type $A_{1}$. For $G$ of exceptional type it follows from Remark 3.39 and the tables in [Carter 1985, $\S 13]$ that there are no nilpotent orbits in $\mathfrak{g}$ that are both spherical and distinguished.

4.2. Orthogonal simple roots and spherical nilpotent orbits. Panyushev [1999, Theorem 3.4] proved that if the characteristic of $k$ is zero, then $e \in \mathcal{N}$ is spherical if and only if there exist pairwise orthogonal simple roots $\alpha_{1}, \alpha_{2}, \ldots, \alpha_{t}$ in $\Pi$ such that $G \cdot e$ contains an element of the form $\sum_{i=1}^{t} e_{\alpha_{i}}$ where $e_{\alpha_{i}} \in \mathfrak{g}_{\alpha_{i}} \backslash\{0\}$. By pairwise orthogonal we mean that $\left\langle\alpha_{i}, \alpha_{j}\right\rangle=0$ for $i \neq j$. In this section we show that this is also the case if the characteristic of $k$ is $\operatorname{good}$ for $G$.

Lemma 4.7. Let $\mathscr{D} G$ be of type $A_{1}^{t}$ for some $t \geqslant 1$. Then there is precisely one distinguished nilpotent orbit in $\mathcal{N}$.

Proof. Since the nilpotent orbits of $G$ in $\mathfrak{g}$ are precisely the nilpotent orbits of $\mathscr{D} G$ in Lie $\mathscr{D} G$, we may assume that $G$ is semisimple. Thus, $G=G_{1} G_{2} \cdots G_{r}$ and each $G_{i}$ is of type $A_{1}$. There is precisely one distinguished nilpotent orbit when $G_{i}$ is of type $A_{1}$ : the unique nonzero nilpotent orbit. Also $G \cdot e$ is distinguished in $\mathfrak{g}$ if and only if $G_{i} \cdot e_{i}$ is distinguished in $\mathfrak{g}_{i}=\operatorname{Lie} G_{i}$ for all $i$, where $e=e_{1}+\cdots+e_{r}$ and $e_{i} \in \mathfrak{g}_{i}$ is nilpotent.

Lemma 4.8. Let $e \in \mathcal{N}$ and $S$ be a maximal torus of $C_{G}(e)$. Then $\mathscr{D} C_{G}(S)$ is of type $A_{1}^{t}$ for some $t \geqslant 1$ if and only if there exist pairwise orthogonal simple roots $\alpha_{1}, \alpha_{2}, \ldots, \alpha_{t}$ in $\Pi$ such that $G \cdot$ e contains an element of the form $\sum_{i=1}^{t} e_{\alpha_{i}}$, where $e_{\alpha_{i}} \in \mathfrak{g}_{\alpha_{i}} \backslash\{0\}$.

Proof. Suppose that $\mathscr{D} C_{G}(S)$ is of type $A_{1}^{t}$. Let $\alpha_{1}, \ldots, \alpha_{t}$ be simple roots of $\Phi$, where $\Phi$ is the root system of $C_{G}(S)$ relative to a maximal torus $T$ of $C_{G}(S)$. As $\mathscr{D} C_{G}(S)$ is of type $A_{1}^{t}$, the roots $\alpha_{1}, \ldots, \alpha_{t}$ are pairwise orthogonal. Clearly,

$$
e \in \operatorname{Lie} C_{G}(S)=\mathfrak{c}_{\mathfrak{g}}(S)
$$

and $e$ is distinguished in $\mathfrak{c}_{\mathfrak{g}}(S)$; see Proposition 2.15. By Lemma 4.7, an element of the form $\sum_{i=1}^{t} e_{\alpha_{i}}$ is also distinguished in $\mathfrak{c}_{\mathfrak{g}}(S)$ and there is precisely one distinguished nilpotent orbit in $\mathfrak{c}_{\mathfrak{g}}(S)$. Thus, $e$ and $\sum_{i=1}^{t} e_{\alpha_{i}}$ are in the same $C_{G}(S)$-orbit; hence they are in the same $G$-orbit. So $G \cdot e$ contains an element of the desired form. 
Conversely, suppose that there exist pairwise orthogonal simple roots $\alpha_{1}, \alpha_{2}$, $\ldots, \alpha_{t} \in \Psi$ such that $G \cdot e$ contains an element of the form $e^{\prime}=\sum_{i=1}^{t} e_{\alpha_{i}}$. Let $H$ be the subgroup of $G$ generated by $\left\{T, U_{ \pm \alpha_{i}} \mid 1 \leqslant i \leqslant t\right\}$, where $T$ is as in the previous paragraph. Then $\mathscr{D} H$ is of type $A_{1}^{t}$. By construction, $e^{\prime}$ is distinguished in $\mathfrak{h}$. By Proposition 2.15, $H$ is of the form $C_{G}\left(S^{\prime}\right)$, where $S^{\prime}$ is a maximal torus of $C_{G}\left(e^{\prime}\right)$. Thus, $\mathscr{D} C_{G}\left(S^{\prime}\right)$ is of type $A_{1}^{t}$. Since $e$ and $e^{\prime}$ are $G$-conjugate, so are $C_{G}(e)$ and $C_{G}\left(e^{\prime}\right)$, as well as $S$ and $S^{\prime}$. Finally, we get that $C_{G}(S)$ and $C_{G}\left(S^{\prime}\right)$ are $G$-conjugate. The result follows.

Lemma 4.9. If $e \in \mathcal{N}$ is spherical, then $\mathscr{D} C_{G}(S)$ is of type $A_{1}^{t}$ for some $t \geqslant 1$.

Proof. Let $\lambda$ be a cocharacter of $G_{G}(S)$ that is associated to $e$, that is, $\lambda \in \Omega_{C_{G}(S)}^{a}(e)$. Then, since Lie $C_{G}(S)=\mathfrak{c}_{\mathfrak{g}}(S)$, it follows from [Fowler and Röhrle 2008, Corollary 3.21] that $\lambda \in \Omega_{G}^{a}(e)$. As $e$ is spherical in $\mathfrak{g}$, we have $\mathrm{ht}(e) \leqslant 3$, by Theorem 3.38. As $\lambda \in \Omega_{C_{G}(S)}^{a}(e)$, we also have $h t(e) \leqslant 3$ when we regard $e$ as an element of $\mathfrak{c}_{\mathfrak{g}}(S)$. Thus, again by Theorem 3.38,e is spherical in $\mathfrak{c}_{\mathfrak{g}}(S)$. So $e$ is distinguished and spherical in $\mathfrak{c}_{\mathfrak{g}}(S)$ and so $\mathscr{D} C_{G}(S)$ is of type $A_{1}^{t}$, by Theorem 4.6.

In order to prove the reverse implication of Lemma 4.9 we first need to consider the group $C_{G}(S)$. If $G$ is classical, then the structure of $C_{G}(S)$ can be determined from the partition $\pi_{e}$ corresponding to $e$; see [Jantzen 2004, §4.8] for the following result.

Lemma 4.10. Let $G$ be simple classical and $e \in \mathcal{N}$ with corresponding partition $\pi_{e}$.

(i) If $G$ is of type $A_{n}$ and $\pi_{e}=\left[1^{r_{1}}, 2^{r_{2}}, \ldots\right]$, then $\mathscr{D} C_{G}(S)$ is of type $\prod_{i \geqslant 1} A_{i-1}^{r_{i}}$.

(ii) If $G$ is of type $B_{n}$ and $\pi_{e}=\left[1^{2 s_{1}+\epsilon_{1}}, 2^{2 s_{2}}, 3^{2 s_{3}+\epsilon_{3}}, \ldots\right]$, where $s_{i} \geqslant 0$ and $\epsilon_{i} \in\{0,1\}$, then $\mathscr{D} C_{G}(S)$ is of type $\prod_{i \geqslant 1} A_{i-1}^{s_{i}} \times B_{m}$, where $2 m+1=\sum_{\epsilon_{i} \neq 0} i$.

(iii) If $G$ is of type $C_{n}$ and $\pi_{e}=\left[1^{2 s_{1}}, 2^{2 s_{2}+\epsilon_{2}}, 3^{2 s_{3}}, 4^{2 s_{4}+\epsilon_{4}}, \ldots\right]$, where $s_{i} \geqslant 0$ and $\epsilon_{i} \in\{0,1\}$, then $\mathscr{D} C_{G}(S)$ is of type $\prod_{i \geqslant 1} A_{i-1}^{s_{i}} \times C_{m}$, where $2 m=\sum_{\epsilon_{i} \neq 0} i$.

(iv) If $G$ is of type $D_{n}$ and $\pi_{e}=\left[1^{2 s_{1}+\epsilon_{1}}, 2^{2 s_{2}}, 3^{2 s_{3}+\epsilon_{3}}, \ldots\right]$, where $s_{i} \geqslant 0$ and $\epsilon_{i} \in\{0,1\}$, then $\mathscr{D} C_{G}(S)$ is of type $\prod_{i \geqslant 1} A_{i-1}^{s_{i}} \times D_{m}$, where $2 m=\sum_{\epsilon_{i} \neq 0} i$.

Lemma 4.11. If $G$ is simple classical and $\mathscr{D} C_{G}(S)$ is of type $A_{1}^{t}$, then e is spherical.

Proof. First suppose that $G$ is of type $A_{n}$. Since $\mathscr{D} C_{G}(S)$ is of type $A_{1}^{t}$, it follows from Lemma 4.10 that $r_{i}=0$ for all $i \geqslant 3$. Thus $\pi_{e}=\left[1^{r_{1}}, 2^{r_{2}}\right]$ and so $e$ is spherical, by Remark 3.39.

Let $G$ be of type $B_{n}$. Since $\mathscr{D} C_{G}(S)$ is of type $A_{1}^{t}$, it follows from Lemma 4.10 that $s_{i}=0$ for $i \geqslant 3$ and $m \leqslant 1$, so $2 m+1 \leqslant 3$. Since $2 m+1$ is a sum of distinct odd integers, we either have $2 m+1=1$ or $2 m+1=3$. Thus $\pi_{e}=\left[1^{2 s_{1}+1}, 2^{2 s_{2}}\right]$ or $\pi_{e}=\left[1^{2 s_{1}}, 2^{2 s_{2}}, 3\right]$ and so $e$ is spherical, again by Remark 3.39 . 
Let $G$ be of type $C_{n}$. Since $\mathscr{D} C_{G}(S)$ is of type $A_{1}^{t}$, it follows from Lemma 4.10 that $s_{i}=0$ for $i \geqslant 3$ and $m \leqslant 1$, so $2 m \leqslant 2$. Since $2 m$ is a sum of distinct even integers, we either have $2 m=0$ or $2 m=2$. Thus $\pi_{e}=\left[1^{2 s_{1}}, 2^{2 s_{2}}\right]$ or $\pi_{e}=$ $\left[1^{2 s_{1}}, 2^{2 s_{2}+1}\right]$ and so, by Remark 3.39, $e$ is spherical.

Finally, let $G$ be of type $D_{n}$. Since $\mathscr{D} C_{G}(S)$ is of type $A_{1}^{t}$, it again follows from Lemma 4.10 that $s_{i}=0$ for $i \geqslant 3$ and $m \leqslant 2$, so $2 m \leqslant 4$. Since $2 m$ is a sum of distinct odd integers, we either have $2 m=0$ or $2 m=1+3$. Thus $\pi_{e}=\left[1^{2 s_{1}}, 2^{2 s_{2}}\right]$ or $\pi_{e}=\left[1^{2 s_{1}+1}, 2^{2 s_{2}}, 3\right]$ and so, by Remark 3.39, $e$ is spherical.

All that remains is to check the exceptional cases. The Bala-Carter label of $e \in \mathcal{N}$ gives the Dynkin type of a Levi subgroup $L$ of $G$ such that $e$ is distinguished in Lie $\mathscr{D} L$. By Proposition 2.15, such a Levi subgroup is the centralizer of a maximal torus of $C_{G}(e)$. Thus, the Bala-Carter label gives the type of $\mathscr{D} C_{G}(S)$. It follows from the tables in [Carter 1985, §13] and Remark 3.39 that any nilpotent orbit with Bala-Carter label $A_{1}^{t}$ is spherical. We summarize this in Table 5.

\begin{tabular}{ccc|ccc} 
Type & Bala-Carter label & Height & Type & Bala-Carter label & Height \\
\hline$G_{2}$ & $A_{1}$ & 2 & $E_{7}$ & $A_{1}$ & 2 \\
& $\widetilde{A_{1}}$ & 3 & & $2 A_{1}$ & 2 \\
$F_{4}$ & $A_{1}$ & 2 & & $\left(3 A_{1}\right)^{\prime \prime}$ & 2 \\
& $\widetilde{A_{1}}$ & 2 & & $\left(3 A_{1}\right)^{\prime}$ & 3 \\
& $A_{1}+\widetilde{A_{1}}$ & 3 & & $4 A_{1}$ & 3 \\
$E_{6}$ & $A_{1}$ & 2 & $E_{8}$ & $A_{1}$ & 2 \\
& $2 A_{1}$ & 2 & & $2 A_{1}$ & 2 \\
& $3 A_{1}$ & 3 & & $3 A_{1}$ & 3 \\
& & & & $4 A_{1}$ & 3
\end{tabular}

Table 5. Orbits in exceptional Lie algebras with $\mathscr{D} C_{G}(S)$ of Type $A_{1}^{t}$.

Lemma 4.12. If $G$ is a simple exceptional algebraic group and $\mathscr{D} C_{G}(S)$ is of type $A_{1}^{t}$, then e is spherical.

Lemma 4.13. Let $e \in \mathcal{N}$. If $\mathscr{D} C_{G}(S)$ is of type $A_{1}^{t}$, then $e \in \mathfrak{g}$ is spherical.

Proof. For $G$ simple, the result follows from Lemmas 4.11 and 4.12. In the general case let $\mathscr{D} G=G_{1} G_{2} \cdots G_{r}$ be a commuting product of simple groups and $e=$ $e_{1}+e_{2}+\cdots+e_{r}$, where $e_{i} \in \mathfrak{g}_{i}=\operatorname{Lie} G_{i}$ and each $e_{i}$ is nilpotent. A maximal torus $S$ of $C_{G}(e)$ is of the form $S_{1} S_{2} \cdots S_{r}$, where $S_{i}$ is a maximal torus of $C_{G_{i}}\left(e_{i}\right)$. The simple case implies that $\mathscr{D} C_{G_{i}}\left(S_{i}\right)$ is of type $A_{1}^{t}$.

Lemmas 4.13 and 4.8 now imply the main result of this section.

Theorem 4.14. Let $e \in \mathcal{N}$ and let $S$ be a maximal torus of $C_{G}(e)$. Then the following are equivalent. 
(i) e is spherical.

(ii) $\mathscr{D C}_{G}(S)$ is of type $A_{1}^{t}$.

(iii) There exist pairwise orthogonal simple roots $\alpha_{1}, \alpha_{2}, \ldots, \alpha_{t} \in \Pi$ such that $G \cdot e$ contains an element of the form $\sum_{i=1}^{t} e_{\alpha_{i}}$, where $e_{\alpha_{i}} \in \mathfrak{g}_{\alpha_{i}} \backslash\{0\}$.

4.3. Spherical orbits and ad-nilpotent ideals. In this section we generalize some results from [Panyushev and Röhrle 2001; 2005] to a field of good characteristic.

When $G$ is simple and classical, Panyushev [1994, §4] gave simple algebraic criteria for a nilpotent element $e \in \mathcal{N}$ to be spherical. We show that these criteria are still valid for a field of good characteristic.

Lemma 4.15. Let $G$ be a simple classical algebraic group and $e \in \mathcal{N}$.

(i) Let e be a nilpotent matrix in $\mathfrak{s l}_{n}$ or $\mathfrak{s p}_{n}$. Then e is spherical if and only if $e^{2}=0$.

(ii) Let e be a nilpotent matrix in $\mathfrak{s o}_{n}$. Then $e$ is spherical if and only if the rank of $e^{2}$ is at most one.

Proof. Let $e$ be a nilpotent matrix in $\mathfrak{s l}_{n}$ or $\mathfrak{s p}_{n}$. If $e$ is spherical, then $\pi_{e}=\left[1^{j}, 2^{i}\right]$, for appropriate $i$ and $j$; see Remark 3.39. By considering the corresponding Jordan blocks for $\pi_{e}$, we see that $e^{2}=0$. Conversely, if $e^{2}=0$, then $e$ is conjugate to an element $e^{\prime}$ with partition $\pi_{e^{\prime}}=\left[1^{j}, 2^{i}\right]$ and so $e$ is spherical, again by Remark 3.39.

Let $e$ be a nilpotent matrix in $\mathfrak{s o}_{n}$. If $e$ is spherical, then $\pi_{e}=\left[1^{j}, 2^{i}\right]$ or $\pi_{e}=\left[1^{j}, 2^{i}, 3\right]$, for appropriate $i$ and $j$; see Remark 3.39. By considering the corresponding Jordan blocks for $\pi_{e}$, we see that either $e^{2}=0$ or $e^{2}$ has partition $\pi_{e^{2}}=\left[1^{k}, 2\right]$. Thus the rank of $e^{2}$ is either 0 or 1 . Conversely, if the rank of $e^{2}$ is at most 1 , then $e$ is conjugate to an element $e^{\prime}$ with partition $\pi_{e^{\prime}}=\left[1^{j}, 2^{i}\right]$ or $\pi_{e^{\prime}}=\left[1^{j}, 2^{i}, 3\right]$ and so $e$ is spherical, again by Remark 3.39.

Panyushev and Röhrle [2001; 2005] gave a classification of the spherical ideals of $\mathfrak{b}=$ Lie $B$ contained in $\mathfrak{b}_{u}=\operatorname{Lie} R_{u}(B)$, where $B$ is a Borel subgroup of $G$ in characteristic 0 . An ideal $\mathfrak{c}$ of $\mathfrak{b}$ is ad-nilpotent if $\mathfrak{c}$ is contained in $\mathfrak{b}_{u}$. An adnilpotent ideal $\mathfrak{c}$ of $\mathfrak{b}$ is called spherical if its $G$-saturation $G \cdot \mathfrak{c}=\{x \cdot e \mid x \in G, e \in \mathfrak{c}\}$ is a spherical $G$-variety. First in [Panyushev and Röhrle 2001, Corollary 2.4] it is proved that if $\mathfrak{a}$ is an abelian ideal of $\mathfrak{b}$, then $\mathfrak{a}$ is spherical. In [Panyushev and Röhrle 2005, Proposition 4.1 and Theorem 4.2] it is proved that there are nonabelian spherical ideals only if $G$ is not simply-laced, that is, if the Dynkin diagram of $G$ has a multiple bond.

[Panyushev and Röhrle 2001, Theorem 2.3] states that any $G$-orbit meeting an abelian ad-nilpotent ideal $\mathfrak{a}$ is spherical. This is proved by means of the fact that an orbit $G \cdot e$ is spherical if and only if $\operatorname{ad}(e)^{4}=0$; see [Panyushev 1994, Corollary 2.2]. 
Unfortunately, this equivalence is no longer true in positive characteristic; see Example 4.17. However, the forward implication of this equivalence is still valid in good characteristic.

Lemma 4.16. If $e \in \mathcal{N}$ is spherical, then $\operatorname{ad}(e)^{4}=0$.

Proof. If $e$ is spherical, then by Theorem 3.38, ht $(e) \leqslant 3$. Let $\mathfrak{g}=\bigoplus_{i=-3}^{3} \mathfrak{g}(i)$ be the grading of $\mathfrak{g}$ afforded by an associated cocharacter in $\Omega_{G}^{a}(e)$. We have that $e \in \mathfrak{g}(2)$. Consequently, $\operatorname{ad}(e)^{4}(\mathfrak{g}(i)) \subseteq \mathfrak{g}(i+8)=\{0\}$ for any $-3 \leqslant i \leqslant 3$. So $\operatorname{ad}(e)^{4}=0$ on all of $\mathfrak{g}$.

The next example shows that the converse of Lemma 4.16 is not true in general in positive characteristic.

Example 4.17. Let $G=\mathrm{SL}_{3}(k)$ and char $k=3$. So $\mathfrak{g}=\mathfrak{s l}_{3}(k)$. Set $e=e_{2,1}+e_{3,2}$, where $e_{i, j}$ is the elementary matrix with a 1 in the $(i, j)$ position and 0 's elsewhere. So $e$ is a regular nilpotent element in $\mathfrak{g}$. Consider the grading of $\mathfrak{g}$ afforded by an associated cocharacter in $\Omega_{G}^{a}(e)$. We have $\mathfrak{g}=\bigoplus_{i=-2}^{2} \mathfrak{g}(2 i)$. In order to prove $\operatorname{ad}(e)^{4}=0$, it is sufficient to show that $\operatorname{ad}(e)^{4}(\mathfrak{g}(-4))=\{0\}$. Clearly, $\mathfrak{g}(-4)=k e_{1,3}$. Now

$$
\operatorname{ad}(e)\left(e_{1,3}\right)=e_{2,3}-e_{1,2} \quad \text { and } \quad \operatorname{ad}(e)\left(e_{2,3}-e_{1,2}\right)=e_{1,1}-2 e_{2,2}+e_{3,3} .
$$

Since char $k=3$, we have

$$
e_{1,1}-2 e_{2,2}+e_{3,3}=e_{1,1}+e_{2,2}+e_{3,3} \quad \text { and } \quad e_{1,1}+e_{2,2}+e_{3,3} \in Z(\mathfrak{g}) .
$$

Thus, $\operatorname{ad}(e)^{4}=0$. However, $e$ is not spherical, as $\pi_{e}=$ [3]; see Remark 3.39.

We note that [Panyushev and Röhrle 2005, Proposition 4.1 and Theorem 4.2] both also hold in good characteristic, as their proofs only require properties of the underlying root system $\Psi$ and the results established in Lemmas 4.15 and 4.16.

So we are left to show that if $\mathfrak{a}$ is an abelian ad-nilpotent ideal, then $\mathfrak{a}$ is spherical. Since $G \cdot \mathfrak{a}$ is irreducible, it is the closure of some nilpotent orbit, say $\overline{G \cdot e}=G \cdot \mathfrak{a}$. The maximal abelian ad-nilpotent ideals of $\mathfrak{b}$ are the same in good characteristic as in characteristic zero; see [Röhrle 1998, Table 1] and [Panyushev and Röhrle 2001, Tables I and II, §4]. Using the description of the orbits in [Panyushev and Röhrle 2001, Tables I and II, §4], we infer that the Bala-Carter label of $G \cdot e$ is of the form $A_{1}^{t}$, so $G \cdot e$ is spherical, thanks to Theorem 4.14. Since $G \cdot e$ is open in $G \cdot \mathfrak{a}$, it follows that $G \cdot \mathfrak{a}$ is spherical. It is straightforward to get the sphericity of $G \cdot \mathfrak{a}$ for any abelian ideal $\mathfrak{a}$ of $\mathfrak{b}$ from the sphericity result of the maximal abelian ideals. Thus we have established the following.

Theorem 4.18. Let $\mathfrak{a}$ be an abelian ad-nilpotent ideal of $\mathfrak{b}$. Then $\mathfrak{a}$ is spherical.

As a corollary of Theorem 4.18 we get [Röhrle 1998, Theorem 1.1] in good characteristic. 
Corollary 4.19. Let $P$ be a parabolic subgroup of $G$ and let $\mathfrak{a}$ be an abelian ideal of Lie $P$ in Lie $R_{u}(P)$. Then $P$ acts on $\mathfrak{a}$ with finitely many orbits.

Remark 4.20. We note that Theorem 4.18 and Corollary 4.19 do in fact hold in arbitrary characteristic; see [Röhrle 1998, Theorem 1.1].

Remark 4.21. If $\mathfrak{c}$ is a spherical ideal of $\mathfrak{b}$, then clearly $B$ acts on $\mathfrak{c}$ with a finite number of orbits. However, the converse does not hold. There are many additional instances when $B$ acts on a given ideal $\mathfrak{c}$ of $\mathfrak{b}$ only with a finite number of orbits (see the results in [Hille and Röhrle 1999; Jürgens and Röhrle 2002]).

4.4. A geometric characterization of spherical orbits. We will now describe a formula characterizing spherical $G$-orbits in a simple algebraic group $G$ in terms of elements of the Weyl group $W$ of $G$ that is proved in [Cantarini et al. 2005, Theorem 1]. For $x \in G$ the conjugacy class $G \cdot x$ is spherical if $G \cdot x$ is a spherical variety. While this characterization in the place cited is based on case-by-case arguments, recently, G. Carnovale [2006, Theorem 2] gave a proof of this result which is free of case-by-case considerations and applies in good odd characteristic. Using the arguments from [Cantarini et al. 2005] combined with our classification of the spherical unipotent nilpotent orbits, Remark 3.40, we can generalize this formula to good characteristic.

Let $G$ be simple and suppose that $p$ is good for $G$. Fix a Borel subgroup $B$ of $G$. Let $W$ be the Weyl group of $G$ and let $B w B$ be the $(B, B)$-double coset of $G$ containing $w \in W$. The following was shown in [Cantarini et al. 2005] in an argument independent of the characteristic of the underlying field: Suppose that 0 is a conjugacy class in $G$ which intersects the double coset $B w B$ so that

$$
\operatorname{dim} 0=\ell(w)+\operatorname{rk}(1-w)
$$

holds. Then $\mathbb{O}$ is spherical. Here $\operatorname{rk}(1-w)$ denotes the rank of the linear map $1-w$ in the standard representation of $W$ and $\ell$ is the usual length function of $W$ with respect to a distinguished set of generators of $W$. Conversely, let 0 be a spherical conjugacy class in $G$ and let $B w B$ be the $(B, B)$-double coset containing the dense $B$-orbit in 0 . Then $\operatorname{dim} 0=\ell(w)+\operatorname{rk}(1-w)$; see [Carnovale 2006, Theorem 2]. Consequently, this gives a geometric characterization of the spherical conjugacy classes in $G$. For proofs we refer the reader to [Cantarini et al. 2005; Carnovale 2006]. Observe that as a consequence of the finiteness of the Bruhat decomposition of $G$ and the fact that any $(B, B)$-double coset and any conjugacy class of $G$ are irreducible subvarieties of $G$, for a given conjugacy class 0 in $G$ there is a unique $w \in W$ such that $O \cap B w B$ is dense in $\mathcal{O}$.

Theorem 4.22 [Cantarini et al. 2005, Theorem 1]. Let 0 be a conjugacy class in $G$ and let $w \in W$ be such that $\mathcal{O} \cap B w B$ is dense in $\mathcal{O}$. Then $\mathcal{O}$ is spherical if and only if $\operatorname{dim} 0=\ell(w)+\operatorname{rk}(1-w)$. 
4.5. Bad primes and spherical nilpotent orbits. Finally, we briefly discuss the situation when the characteristic of $k$ is bad for $G$. In this case the classification of the nilpotent orbits in $\mathcal{N}$ is different from that in good characteristic; see [Carter 1985, §5.11]. However, there is still only a finite number of nilpotent orbits [Holt and Spaltenstein 1985]. Unfortunately, our methods do not allow us to give a classification of the spherical nilpotent orbits in this case, for in our classification we made use of the height of a nilpotent orbit, where the height is defined via an associated cocharacter. However, it is not known whether associated cocharacters always exist for all nilpotent elements in bad characteristic; see [Jantzen 2004, $\S 5.14, \S 5.15]$.

In principle one can still determine whether a given nilpotent orbit is spherical by a case-by-case analysis. Next we give two examples of this. In particular, we show that Theorem 4.14 fails in bad characteristic in general. These examples show that there can be additional spherical nilpotent orbits in bad characteristic.

Examples 4.23. (i) Let $G$ be of type $B_{2}$ and char $k=2$. Let $\alpha$ and $\beta$ be the simple roots of $\Psi$ with $\alpha$ the long root. Let $e=e_{\alpha+\beta}+e_{\alpha+2 \beta}$. According to [Jantzen $2004, \S 5.14]$ the centralizer $C_{G}(e)$ is the unipotent radical of a Borel subgroup of $G$. Thus, by Lemma 2.11, $C_{G}(e)$ is a spherical subgroup of $G$ and so $e$ is spherical. Note that the $G$-orbit of $e$ does not contain an element of the form $e_{\alpha}$ or $e_{\beta}$, but $e$ is still spherical. Thus, Theorem 4.14 is no longer true in bad characteristic. Moreover, $e$ is distinguished in $\mathfrak{g}$ [Jantzen 2004, §5.14]. This shows that Theorem 4.6 can also fail for bad characteristic.

(ii) Let $G$ be of type $G_{2}$ and char $k=3$. Let $\alpha$ and $\beta$ be the simple roots of $\Psi$ with $\alpha$ the long root. Let $e=e_{\alpha+2 \beta}+e_{2 \alpha+3 \beta}$. According to [Jantzen 2004, §5.15], the centralizer $C_{G}(e)$ is the unipotent radical of a Borel subgroup of $G$. Thus, by Lemma 2.11, $C_{G}(e)$ is a spherical subgroup of $G$ and so $e$ is spherical. Again, the $G$-orbit of $e$ does not contain an element of the form $e_{\alpha}$ or $e_{\beta}$, but $e$ is spherical. Again, $e$ is distinguished in $\mathfrak{g}$ [Jantzen 2004, §5.15].

\section{Acknowledgements}

We are grateful to S. M. Goodwin for providing the relative version DOOBSLevi of his program DOOBS that was used in Section 3.7 to determine the sphericity of the nilpotent orbits of height 3 for the exceptional cases and for very helpful discussions and improvements of the paper. We would also like to thank the referee for suggesting some improvements.

\section{References}

[Azad et al. 1990] H. Azad, M. Barry, and G. Seitz, "On the structure of parabolic subgroups", Comm. Algebra 18:2 (1990), 551-562. MR 91d:20048 Zbl 0717.20029 
[Bardsley and Richardson 1985] P. Bardsley and R. W. Richardson, "Étale slices for algebraic transformation groups in characteristic p", Proc. London Math. Soc. (3) 51:2 (1985), 295-317. MR 86m:14034 Zbl 0604.14037

[Borel 1991] A. Borel, Linear algebraic groups, 2nd ed., Graduate Texts in Mathematics 126, Springer, New York, 1991. MR 92d:20001 Zbl 0726.20030

[Bourbaki 1968] N. Bourbaki, Éléments de mathématique. Fasc. XXXIV. Groupes et algèbres de Lie. Chapitre IV: Groupes de Coxeter et systèmes de Tits. Chapitre V: Groupes engendrés par des réflexions. Chapitre VI: systèmes de racines, Actualités Scientifiques et Industrielles 1337, Hermann, Paris, 1968. MR 39 \#1590 Zbl 0186.33001

[Brion 1986] M. Brion, "Quelques propriétés des espaces homogènes sphériques", Manuscripta Math. 55:2 (1986), 191-198. MR 87g:14054 Zbl 0604.14048

[Brion 1987] M. Brion, "Classification des espaces homogènes sphériques”, Compositio Math. 63:2 (1987), 189-208. MR 89d:32068 Zbl 0642.14011

[Brion 1995] M. Brion, "Spherical varieties", pp. 753-760 in Proceedings of the International Congress of Mathematicians (Zürich, 1994), vol. 2, edited by S. D. Chatterji, Birkhäuser, Basel, 1995. MR 97f:14049 Zbl 0862.14031

[Brundan 1998] J. Brundan, "Dense orbits and double cosets", pp. 259-274 in Algebraic groups and their representations (Cambridge, 1997), edited by R. W. Carter and J. Saxl, NATO Adv. Sci. Inst. Ser. C Math. Phys. Sci. 517, Kluwer Acad. Publ., Dordrecht, 1998. MR 99k:20090 Zbl 0933.20038

[Cantarini et al. 2005] N. Cantarini, G. Carnovale, and M. Costantini, "Spherical orbits and representations of $U_{\epsilon}(\mathfrak{g})$ ", Transform. Groups 10:1 (2005), 29-62. MR 2005m:17020 Zbl 1101.17006

[Carnovale 2006] G. Carnovale, "Spherical conjugacy classes and involutions in the Weyl group", preprint, 2006. To appear in Math. Z. arXiv math/0612408

[Carter 1985] R. W. Carter, Finite groups of Lie type: Conjugacy classes and complex characters, Wiley, New York, 1985. MR 87d:20060 Zbl 0567.20023

[Fowler and Röhrle 2008] R. A. Fowler and G. Röhrle, "On cocharacters associated to nilpotent elements of reductive groups", Nagoya Mathematics Journal 190 (2008), 105-128.

[Gap 2004] The Gap Group, Groups, Algorithms and Programming, Version 4.3, 2004, Available at http://www-gap.dcs.st-and.ac.uk.

[Goodwin 2005a] S. Goodwin, "Algorithmic testing for dense orbits of Borel subgroups", J. Pure Appl. Algebra 197:1-3 (2005), 171-181. MR 2006c:14069 Zbl 1066.14054

[Goodwin 2005b] S. M. Goodwin, "Relative Springer isomorphisms", J. Algebra 290:1 (2005), 266281. MR 2006c:20095 Zbl 02196726

[Goodwin 2006a] S. M. Goodwin, DOOBS and DOOBSLevi programs, available at http://web.mat. bham.ac.uk/S.M.Goodwin/DOOBS/program/program.html

[Goodwin 2006b] S. M. Goodwin, "On the conjugacy classes in maximal unipotent subgroups of simple algebraic groups”, Transform. Groups 11 (2006), 51-76. MR 2006k:20096 Zbl 1118.20041

[Hille and Röhrle 1999] L. Hille and G. Röhrle, "A classification of parabolic subgroups of classical groups with a finite number of orbits on the unipotent radical", Transform. Groups 4:1 (1999), 35-52. MR 2000f:20072 Zbl 0924.20035

[Holt and Spaltenstein 1985] D. F. Holt and N. Spaltenstein, "Nilpotent orbits of exceptional Lie algebras over algebraically closed fields of bad characteristic", J. Austral. Math. Soc. Ser. A 38:3 (1985), 330-350. MR 86g:17007 Zbl 0575.17007

[Humphreys 1972] J. E. Humphreys, Introduction to Lie algebras and representation theory, Graduate Texts in Mathematics 9, Springer, New York, 1972. MR 48 \#2197 Zbl 0254.17004 
[Humphreys 1975] J. E. Humphreys, Linear algebraic groups, Graduate Texts in Mathematics 21, Springer, New York, 1975. MR 53 \#633 Zbl 0325.20039

[Humphreys 1995] J. E. Humphreys, Conjugacy classes in semisimple algebraic groups, Mathematical Surveys and Monographs 43, American Mathematical Society, Providence, RI, 1995. MR 97i:20057 Zbl 0834.20048

[Jantzen 2004] J. C. Jantzen, "Nilpotent orbits in representation theory", pp. 1-211 in Lie theory: Lie algebras and representations, edited by J.-P. Anker and B. Orsted, Progr. Math. 228, Birkhäuser, Boston, 2004. MR 2005c:14055 Zbl 02160654

[Jürgens and Röhrle 2002] U. Jürgens and G. Röhrle, "MOP—algorithmic modality analysis for parabolic group actions”, Experiment. Math. 11:1 (2002), 57-67. MR 2004a:20050 Zbl 1050.20033

[Kempf 1978] G. R. Kempf, "Instability in invariant theory", Ann. of Math. (2) 108:2 (1978), 299316. MR 80c:20057 Zbl 0406.14031

[Knop 1995] F. Knop, “On the set of orbits for a Borel subgroup”, Comment. Math. Helv. 70:2 (1995), 285-309. MR 96c:14039 Zbl 0828.22016

[Krämer 1979] M. Krämer, "Sphärische Untergruppen in kompakten zusammenhängenden Liegruppen”, Compositio Math. 38:2 (1979), 129-153. MR 80f:22011 Zbl 0402.22006

[Lawther 1999] R. Lawther, "Finiteness of double coset spaces", Proc. London Math. Soc. (3) 79:3 (1999), 605-625. MR 2000f:20075 Zbl 1030.20029

[Luna and Vust 1983] D. Luna and T. Vust, "Plongements d'espaces homogènes", Comment. Math. Helv. 58:2 (1983), 186-245. MR 85a:14035 Zbl 0545.14010

[McNinch 2004] G. J. McNinch, "Nilpotent orbits over ground fields of good characteristic", Math. Ann. 329:1 (2004), 49-85. MR 2005j:17018 Zbl 02123994

[Mumford and Fogarty 1982] D. Mumford and J. Fogarty, Geometric invariant theory, 2nd ed., Ergebnisse der Mathematik und ihrer Grenzgebiete 34, Springer, Berlin, 1982. MR 86a:14006 Zbl 0504.14008

[Panyushev 1990] D. I. Panyushev, "Complexity and rank of homogeneous spaces", Geom. Dedicata 34:3 (1990), 249-269. MR 92e:14046 Zbl 0706.14032

[Panyushev 1994] D. I. Panyushev, "Complexity and nilpotent orbits", Manuscripta Math. 83:3-4 (1994), 223-237. MR 95e:14039 Zbl 0822.14024

[Panyushev 1999] D. I. Panyushev, "On spherical nilpotent orbits and beyond", Ann. Inst. Fourier (Grenoble) 49:5 (1999), 1453-1476. MR 2000i:14072 Zbl 0944.17013

[Panyushev and Röhrle 2001] D. Panyushev and G. Röhrle, "Spherical orbits and abelian ideals", Adv. Math. 159:2 (2001), 229-246. MR 2002c:20073 Zbl 0993.22016

[Panyushev and Röhrle 2005] D. Panyushev and G. Röhrle, "On spherical ideals of Borel subalgebras”, Arch. Math. (Basel) 84:3 (2005), 225-232. MR 2005k:14100 Zbl 1076.14061

[Parshin and Shafarevich 1994] A. N. Parshin and I. R. Shafarevich (editors), Algebraic geometry, IV, Encyclopaedia of Mathematical Sciences 55, Springer, Berlin, 1994. MR 95g:14002 Zbl 0788.00015

[Premet 2003] A. Premet, "Nilpotent orbits in good characteristic and the Kempf-Rousseau theory", J. Algebra 260:1 (2003), 338-366. MR 2004i:17014 Zbl 1020.20031

[Richardson 1967] R. W. Richardson, Jr., "Conjugacy classes in Lie algebras and algebraic groups", Ann. of Math. (2) 86 (1967), 1-15. MR 36 \#173 Zbl 0153.04501

[Richardson 1977] R. W. Richardson, "Affine coset spaces of reductive algebraic groups", Bull. London Math. Soc. 9:1 (1977), 38-41. MR 55 \#10473 Zbl 0355.14020 
[Richardson 1982] R. W. Richardson, "On orbits of algebraic groups and Lie groups", Bull. Austral. Math. Soc. 25:1 (1982), 1-28. MR 83i:14041 Zbl 0467.14008

[Richardson et al. 1992] R. Richardson, G. Röhrle, and R. Steinberg, "Parabolic subgroups with abelian unipotent radical", Invent. Math. 110:3 (1992), 649-671. MR 93j:20092 Zbl 0786.20029

[Röhrle 1998] G. Röhrle, "On normal abelian subgroups in parabolic groups", Ann. Inst. Fourier (Grenoble) 48:5 (1998), 1455-1482. MR 99i:20062 Zbl 0933.20034

[Rousseau 1978] G. Rousseau, "Immeubles sphériques et théorie des invariants", C. R. Acad. Sci. Paris Sér. A-B 286:5 (1978), A247-A250. MR 58 \#22063 Zbl 0375.14013

[Seitz 1998] G. M. Seitz, "Double cosets in algebraic groups", pp. 241-257 in Algebraic groups and their representations (Cambridge, 1997), edited by R. W. Carter and J. Saxl, NATO Adv. Sci. Inst. Ser. C Math. Phys. Sci. 517, Kluwer Acad. Publ., Dordrecht, 1998. MR 99k:20096 Zbl 0933.20039

[Springer 1985] T. A. Springer, "Some results on algebraic groups with involutions", pp. 525-543 in Algebraic groups and related topics (Kyoto/Nagoya, 1983), edited by R. Hotta, Adv. Stud. Pure Math. 6, North-Holland, Amsterdam, 1985. MR 86m:20050 Zbl 0628.20036

[Springer 1998] T. A. Springer, Linear algebraic groups, 2nd ed., Progress in Mathematics 9, Birkhäuser, Boston, 1998. MR 99h:20075 Zbl 0927.20024

[Springer and Steinberg 1970] T. A. Springer and R. Steinberg, "Conjugacy classes", pp. 167-266 in Seminar on algebraic groups and related finite groups (Princeton, N.J., 1968/69), Lecture Notes in Mathematics 131, Springer, Berlin, 1970. MR 42 \#3091 Zbl 0249.20024

[Vinberg 1986] È. B. Vinberg, "Complexity of actions of reductive groups", Funktsional. Anal. $i$ Prilozhen. 20:1 (1986), 1-13, 96. MR 87j:14077 Zbl 0601.14038

Received December 7, 2007. Revised May 27, 2008.

\section{RUSSELL FOWLER}

SCHOOL OF MATHEMATICS

UNIVERSITY OF BIRMINGHAM

BIRMINGHAM B15 2TT

UNITED KINGDOM

fowlerra@maths.bham.ac.uk

GERHARD RÖHRLE

FAKULTÄT FÜR MATHEMATIK

RUHR-UNIVERSITÄT BOCHUM

UNIVERSITÄTSSTRASSE 150

44780 BOCHUM

GERMANY

gerhard.roehrle@rub.de

http://www.ruhr-uni-bochum.de/ffm/Lehrstuehle/Lehrstuhl-VI/rubroehrle.html 